\title{
Could drugs inhibiting the mevalonate pathway also target cancer stem cells?
}

\author{
Wirginia Likus ${ }^{a}$, Krzysztof Siemianowicz ${ }^{b}$, Konrad Bieńk ${ }^{c}$, Małgorzata Pakuła ${ }^{d}$, \\ Himani Pathak ${ }^{\mathrm{e}}$, Chhanda Dutta ${ }^{\mathrm{f}}$, Qiong Wang ${ }^{\mathrm{g}}$, Shahla Shojaei ${ }^{\mathrm{h}}$, Yehuda G. Assaraf ${ }^{\mathrm{i}}$, \\ Saeid Ghavami ${ }^{\mathrm{j}, \mathrm{k}}$, Artur Cieślar-Pobuda ${ }^{1}$, Marek J. Łos ${ }^{\mathrm{m}, \mathrm{n}, *}$
}

\author{
a Department of Human Anatomy, School of Medicine in Katowice, Medical University of Silesia, 18 Medyków Street, 40-752 Katowice, Poland \\ ${ }^{\mathrm{b}}$ Department of Biochemistry, School of Medicine in Katowice, Medical University of Silesia, 18 Medyków Street, 40-752 Katowice, Poland \\ ${ }^{c}$ Interdisciplinary Nanoscience Center (iNANO), Aarhus University, DK-8000 Aarhus C, Denmark \\ d Department of Biomedicine, Aarhus University, DK-8000 Aarhus C, Denmark \\ e Indian Institute of Science Education and Research (IISER TVM), Thiruvananthapuram, Kerala, India \\ ${ }^{\mathrm{f}}$ Department of Clinical \&' Experimental Medicine (IKE), Linköping University, Linköping, Sweden \\ ${ }^{g}$ Department of Physics, Chemistry and Biology (IFM), Division of Biotechnology, Linköping University, Linköping, Sweden \\ ${ }^{\mathrm{h}}$ Department of Clinical Biochemistry, School of Pharmacy and Pharmaceutical Sciences, Isfahan University of Medical Sciences, Isfahan, Iran \\ ${ }^{i}$ The Fred Wyszkowski Cancer Research Laboratory, Department of Biology, Technion-Israel Institute of Technology, Haifa 32000, Israel \\ j Department of Human Anatomy and Cell Science, College of Medicine, Faculty of Health Sciences, University of Manitoba, Winnipeg, MB, Canada \\ ${ }^{\mathrm{k}}$ Health Research Policy Center, Shiraz University of Medical Science, Shiraz, Iran \\ ${ }^{1}$ Institute of Automatic Control, Silesian University of Technology, Gliwice, Poland \\ m LinkoCare Life Sciences AB, 58330 Linköping, Sweden \\ ${ }^{n}$ ENT Department, School of Medicine in Katowice, Medical University of Silesia, 20-24 Francuska Street, 40-027 Katowice, Poland
}

\section{A R T I C L E I N F O}

\section{Article history:}

Received 9 June 2015

Received in revised form

12 December 2015

Accepted 28 January 2016

\section{Keywords:}

Cancer stem-like cells

Mevalonate cascade

Ras

Rho

Stemness

Statins

Yap

\begin{abstract}
A B S T R A C T
Understanding the connection between metabolic pathways and cancer is very important for the development of new therapeutic approaches based on regulatory enzymes in pathways associated with tumorigenesis. The mevalonate cascade and its rate-liming enzyme HMG CoA-reductase has recently drawn the attention of cancer researchers because strong evidences arising mostly from epidemiologic studies, show that it could promote transformation. Hence, these studies pinpoint HMG CoA-reductase as a candidate proto-oncogene. Several recent epidemiological studies, in different populations, have proven that statins are beneficial for the treatment-outcome of various cancers, and may improve common cancer therapy strategies involving alkylating agents, and antimetabolites. Cancer stem cells/cancer initiating cells (CSC) are key to cancer progression and metastasis. Therefore, in the current review we address the different effects of statins on cancer stem cells. The mevalonate cascade is among the most pleiotropic, and highly interconnected signaling pathways. Through G-protein-coupled receptors (GRCP), it integrates extra-, and intracellular signals. The mevalonate pathway is implicated in cell stemness, cell proliferation, and organ size regulation through the Hippo pathway (e.g. Yap/Taz signaling axis). This pathway is a prime preventive target through the administration of statins for the prophylaxis of obesityrelated cardiovascular diseases. Its prominent role in regulation of cell growth and stemness also invokes its role in cancer development and progression. The mevalonate pathway affects cancer metastasis in several ways by: (i) affecting epithelial-to-mesenchymal transition (EMT), (ii) affecting remodeling of
\end{abstract}

Abbreviations: ACAT, acetoacetyl-CoA transferase; acetyl-CoA, acetyl-coenzyme A; Arp2/3, actin-related protein 2/3 (actin polymerizing complex); BPs, bisphospho-

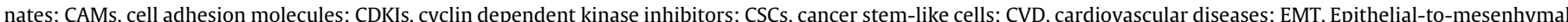

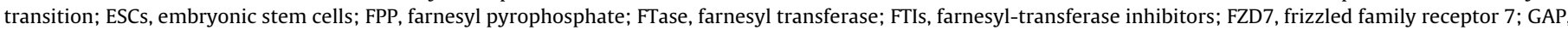

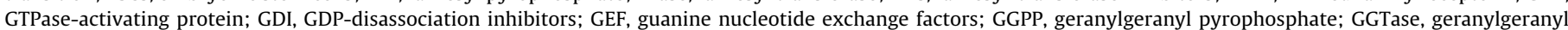

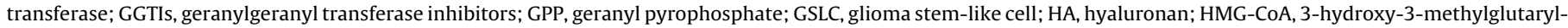

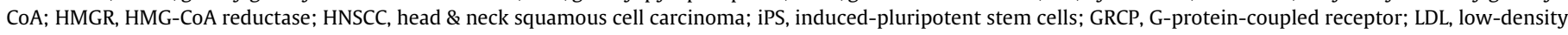

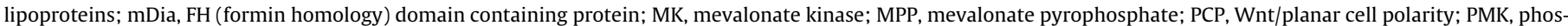

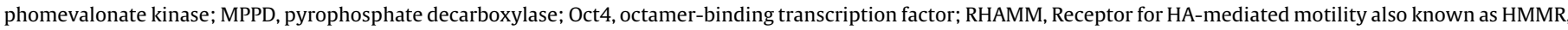

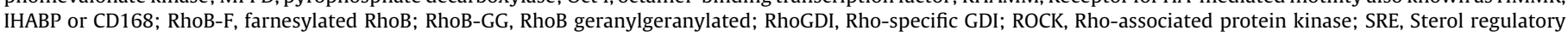
element; SREBP, SRE-binding protein; WAVE, WASP (Wiskott-Aldrich syndrome protein) verprolin homologous; YAP, Yes-associated protein.

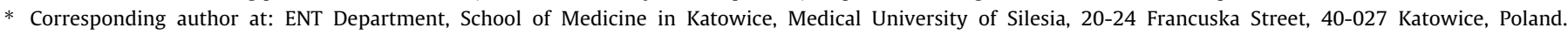
Tel.: +48663288746.

E-mail address: mjelos@gmail.com (M.J. Łos). 
the cytoskeleton as well as cell motility, (iii) affecting cell polarity (non-canonical Wnt/planar pathway), and (iv) modulation of mesenchymal-to-epithelial transition (MET). Herein we provide an overview of the mevalonate signaling network. We then briefly highlight diverse functions of various elements of this mevalonate pathway. We further discuss in detail the role of elements of the mevalonate cascade in stemness, carcinogenesis, cancer progression, metastasis and maintenance of cancer stem cells.

(C) 2016 The Authors. Published by Elsevier Ltd. This is an open access article under the CC BY-NC-ND license (http://creativecommons.org/licenses/by-nc-nd/4.0/).

\section{Introduction}

Statins, competitive inhibitors of 3-hydroxy-3-methylglutarylCoA (HMGCoA) reductase, form the basic class of hypolipidemic drugs used in both primary and secondary prevention of cardiovascular diseases (CVD). CVD are currently the leading cause of death in all Western countries. Nowadays CVD are considered a pandemic. A decrease in death from CVD observed during the last 40 years can be attributed in above $50 \%$ of patients due to a reduction in the intensity of risk factors, e.g. hypercholesterolemia, or their elimination such as smoking cessation (Di Chiara and Vanuzzo, 2009). Among 3 major risk factors, which could be controlled using drugs: hypercholesterolemia, arterial hypertension and obesity, hypercholesterolemia is the best controlled. The goal LDL-cholesterol level is achieved by $55 \%$ of patients, whereas BMI $<25$ is achieved by only $18 \%$ of patients. The best control of lipid disorders in CVD prevention could not be achieved without the use of statins. Nowadays statins are the most frequently used hypolipidemic drugs. They constitute a part of the golden standard "ABS" (aspirin, $\beta$-blocker and statin) in a treatment of patients after myocardial infarction. The introduction of statins was a milestone in CVD prevention (Taylor, 2012).

Osteoporosis is a disease causing bone loss resulting in a decrease in bone mineral density. It may occur in postmenopausal women, elderly people or as a serious side effect of corticosteroid therapy. Bisphosphonates are drugs used in the treatment of osteoporosis to improve mineral bone density and to prevent osteoporotic fractures. The introduction of bisphosphonates has made a great improvement in the treatment of osteoporosis.

There is a growing body of evidence suggesting antitumor activity of statins (Yeganeh et al., 2014). Preclinical studies suggest that bisphosphonates have anticancer activity, in particular nitrogen-containing bisphosphonates have the potential to improve prognosis. Some studies indicate that combination of statin and bisphosphonate can extend the lifespan of experimental animals bearing cancer (Tardoski et al., 2015; Van Acker et al., 2016; Zhao and Hu, 2015; Misra et al., 2015). These two drug classes, although registered to be used in different medical conditions, have also antitumor properties. They have one in common, they interact in the mevalonate pathway. Drug resistance is a serious problem in oncology. Researchers focus their efforts on better understanding of its mechanisms and overcoming it. New antifolates targeting various enzymes are being introduced into clinical use (Gonen and Assaraf, 2012). An intracellular metabolism of these drugs and enzymes involved in it are better understood (Assaraf, 2007; Wojtuszkiewicz et al., 2016). Cancer cells can develop various mechanisms of drug resistance, like fast efflux of anticancer drug mediated by multidrug resistance proteins, drug-accumulating lysosomes and highly acidic microenvironment of tumors (Taylor et al., 2015; Zhitomirsky and Assaraf, 2016). Scientists intensively try to develop new more effective strategies of anticancer therapies. Their efforts are focused either on improvement of efficacy of already existing agents, e.g. introducing nanovehicles enabling targeted delivery of a drug or finding new targets for new anticancer treatment (Livney and Assaraf, 2013). The mevalonate pathway seems to be a very promising new aim for the improvement of efficacy of anticancer pharmacotherapy.
In the current review we discuss the role of the mevalonate pathway focusing on cancer stem cells. We also discuss the role of this pathway in cancer development and anticancer treatment. Furthermore, targeting the mevalonate pathway in cancer, and in CSC in particular, has the potential to overcome anticancer drug resistance, a pleiotropic phenomenon that continues to be a primary hindrance to successful cancer therapy.

\section{Biochemistry of the mevalonate pathway}

The mevalonate pathway, previously known as cholesterol synthesis pathway, is a source of several important biochemical compounds and is implicated in key cellular processes (Yeganeh et al., 2014). This pathway converts acetyl-coenzyme A (acetyl-CoA) to mevalonate in three steps, and further through a series of intermediate steps into farnesyl pyrophosphate (FPP). FPP serves as a precursor in the biosynthesis of sterols including cholesterol, as well as ubiquinone, heme A and dolichols. Furthermore, FPP is converted into geranylgeranyl pyrophosphate (GGPP; Fig. 1). Both FPP and GGPP play a central role in the process of prenylation, where FPP or GGPP are post-translationally added to a protein to facilitate cell membrane anchoring (Ghavami et al., 2012a, 2014).

The pathway is initiated by acetoacetyl-CoA transferase (ACAT), which condenses two acetyl-CoA into acetoacetyl-CoA. Acetoacetyl-CoA is then condensed with an additional acetyl-CoA into 3-hydroxy-3-methylglutaryl-CoA (HMG-CoA) by HMG-CoA synthase. The third step, catalyzed by HMG-CoA reductase (HMGR), converts HMG-CoA to mevalonate. The latter is the rate-limiting step in the mevalonate pathway, and the enzyme HMGR is highly regulated via transcription, phosphorylation and degradation (Goldstein and Brown, 1990). Furthermore, both HMG-CoA synthase and HMGR are regulated by feedback inhibition by the pathway product, cholesterol.

Mevalonate is converted to phosphomevalonate by mevalonate kinase (MK). However, although this step is not rate-limiting, it is highly controlled by feedback inhibition by geranylpyrophosphate, farnesylpyrophosphate and geranylgeranylpyrophosphate (Hinson et al., 1997). This is followed by phosphorylation- and decarboxylation steps, yielding first mevalonate pyrophosphate (MPP) and subsequently isopentenyl-5-pyrophosphate (IPP), and it is catalyzed by phosphomevalonate kinase (PMK) and mevalonate pyrophosphate decarboxylase (MPPD) (Bonetti et al., 2003).

Geranyl pyrophosphate synthase uses IPP and its isomer dimethylallyl-PP to synthesize the 10-carbon geranyl pyrophosphate (GPP). Further chain elongation is carried out by the addition of IPP via farnesyl synthase to yield the 15-carbon FPP. Following the synthesis of FPP, the pathway diverges into several different branches. FPP synthase is therefore a key enzyme as it catalyzes the final common step of the pathway. From FPP, the pathway diverges into numerous branches, mainly GGPP and cholesterol biosynthesis (Fig. 2) (Goldstein and Brown, 1990). The 20-carbon GGPP is synthesized by GGPP synthase from FPP and IPP, and is, together with FPP, an important factor in post-translational protein prenylation (Casey and Seabra, 1996; Novelli and D'Apice, 2012). In addition to its role in prenylation, GGPP is also a precursor for other isoprenoids including ubiquinone (Ghavami et al., 2012b). Along the 


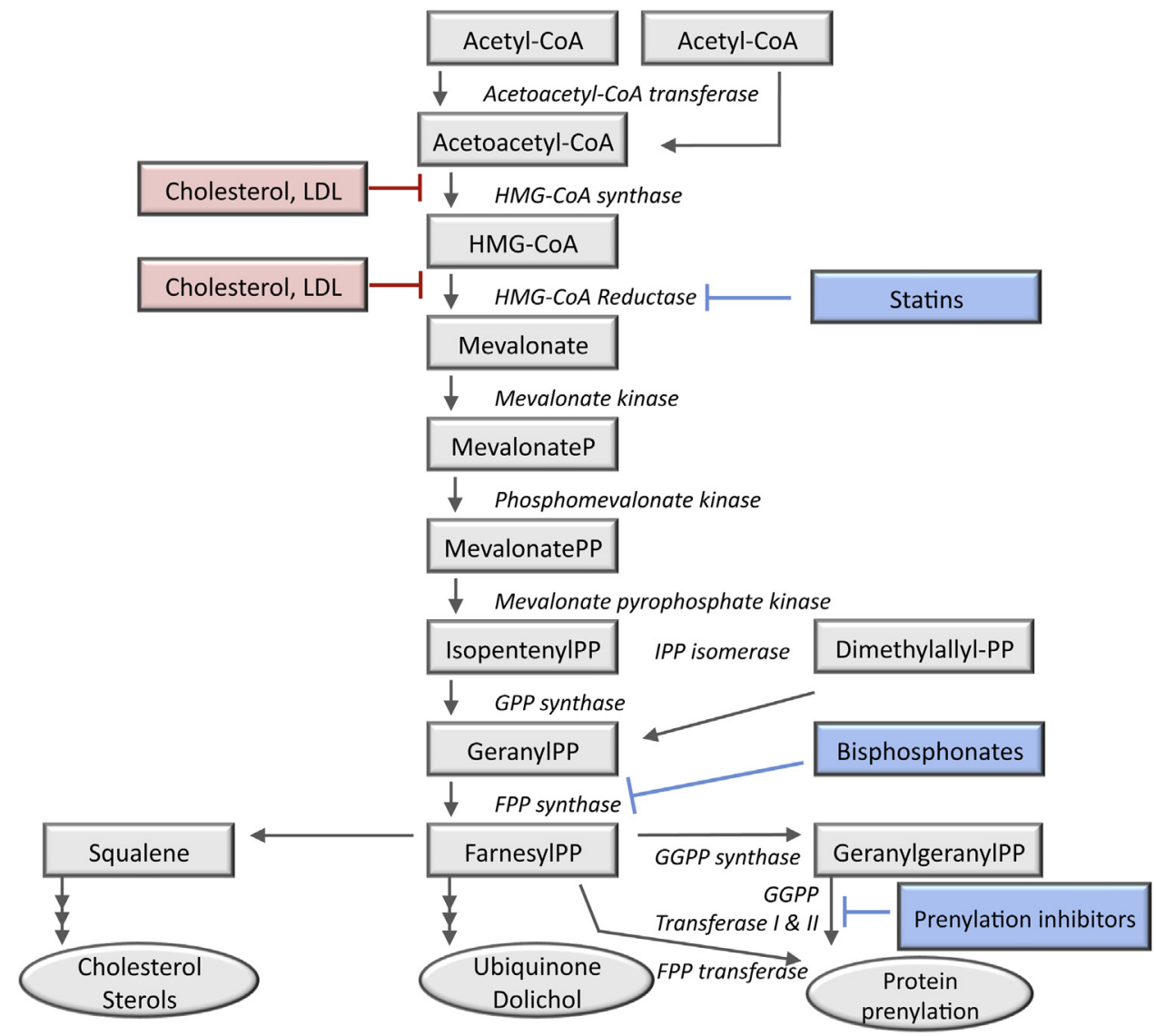

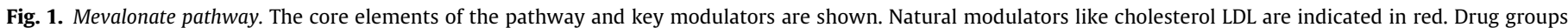
targeting the pathway are indicated in blue. (For interpretation of the references to color in this figure legend, the reader is referred to the web version of this article.)

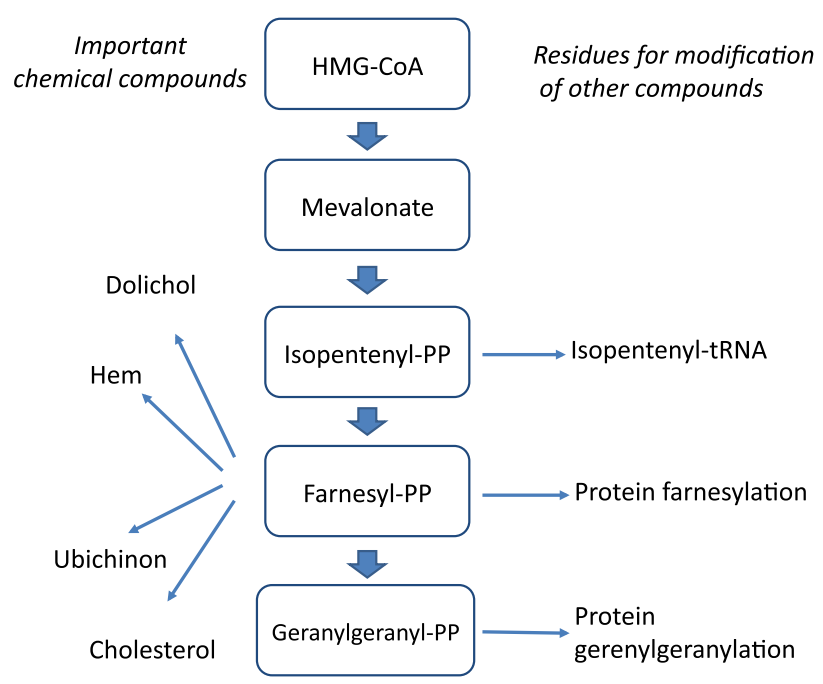

Fig. 2. Important products of mevalonate pathway. The conversion cascade leading to various products used for macromolecule (proteins and tRNA) modification, as well as typical acceptors for the prenyls and their derivatives are shown.

other branch, squalene synthase condenses two FPP resulting in the 30 -carbon squalene. In a series of steps, squalene is converted into cholesterol, which plays an important metabolic role, and is the precursor for a wide range of other molecules including bile acids, steroid hormones, and vitamin D. Cholesterol is also a dominant constituent of cellular membranes in mammalian cells accounting for as much as $25 \%$ of the plasma membrane.

\section{Regulation of the mevalonate signaling pathway}

Due to its central role, the mevalonate pathway is tightly regulated at several steps, as sufficient amounts of cholesterol and isoprenoids are needed for cell growth and proliferation, and since cholesterol is toxic at a higher concentration, cells must maintain a delicate balance of cholesterol biosynthesis. This balance is maintained by the regulation of both cholesterol uptake and biosynthesis, and the main mediator of this regulation is cholesterol itself. In humans, most cholesterol is produced by the liver, and transported via low-density lipoproteins (LDL) to other tissues, and only fast-proliferating cells require cholesterol biosynthesis. Abundance of LDL reduces the activity of HMG-CoA synthase and HMGR to $10 \%$, allowing the cells to maintain only the production of the non-sterol products in the biosynthetic pathway. Both enzymes are regulated by feedback inhibition by cholesterol itself, as well as mevalonate (Goldstein and Brown, 1990; Panini et al., 1989; Brown and Goldstein, 1980).

HMGR is the rate-limiting enzyme in the mevalonate pathway, and therefore it is the most regulated enzyme. In addition to direct inhibition, the enzyme is regulated via control of transcription, translation, phosphorylation and degradation, allowing the cell to adjust activity in response to a range of different stimuli (Burg and Espenshade, 2011). When HMGR is inhibited and mevalonate depleted, cells respond with an up to 200-fold increase in HMGR expression in an attempt to compensate for the loss of activity. This response exemplifies the control of the enzyme, as it is a combination of different mechanisms, all of which result in a large increase in HMGR levels. Sterol regulatory elements (SRE) enhance the transcription of the enzyme in the absence of sterols, and at the same time, an HMGR degradation pathway is inhibited (DeBose-Boyd, 


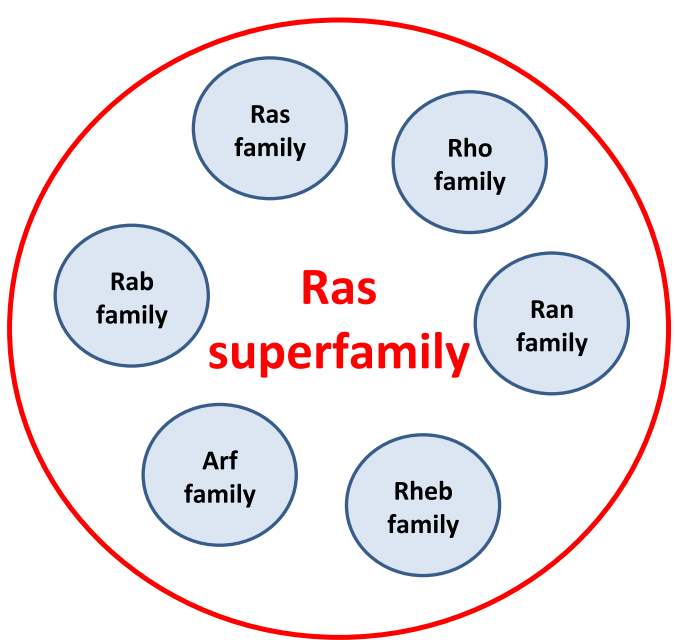

Fig. 3. Ras-superfamily. The subfamilies of Ras-superfamily are shown.

2008). The control of both expression and activity levels of the reductase is very complex. Due to the requirement of non-sterol isoprenoids, even in the presence of large excess of cholesterol and sterols, the pathway must remain active, but must also be able to increase bulk cholesterol production during depletion.

\section{Role of the intermediate components in the mevalonate pathway in metabolism and cell cycle regulation}

The mevalonate pathway is a source of various important biochemicals and residues used for the modification of other compounds and proteins (Fig. 3).

\subsection{Cholesterol}

Cholesterol is a key component essential for the proper function of the cell membrane, and serves as a precursor for the biosynthesis of steroid hormones, bile salts and vitamin D. In addition to dietary intake of cholesterol, it can only be synthesized from squalene, and therefore its biosynthesis is tightly related to, and dependent on the mevalonate pathway. Cholesterol, and its sterol derivatives also function as the main regulatory molecules for the mevalonate pathway by feedback inhibition, and therefore cholesterol has a direct effect on the pathway regulation, and vice versa.

Due to its central role in the cell membrane as well as in the mevalonate pathway, cholesterol plays an important role in regulation of cell cycle progression. Early studies revealed that cholesterol biosynthesis could be inhibited by provision of sterols in the culture medium, which in turn resulted in inhibition of cell growth (Brown and Goldstein, 1974), and that the overall rate of proliferation depends on the cellular levels of cholesterol (Haeffner et al., 1984). Subsequent studies have shown that the total amount of cholesterol varies depending on the phase of the cell cycle, with lower amounts needed in the two G-phases, and that progression to the S-phase requires increased cholesterol levels. Although discrepancy exists regarding the mechanism of cholesterol-mediated cell cycle arrest, there is agreement that cells fail to enter the S-phase when their cholesterol is depleted. In this respect, cells lacking cholesterol are arrested in either the $\mathrm{G}_{1}$ (Keyomarsi et al., 1991; Singh et al., 2013), or $\mathrm{G}_{2}$ phase (Haeffner et al., 1984; Fernandez et al., 2004) until a new source of cholesterol or mevalonate becomes available. Inhibition of the mevalonate pathway could therefore be used to control and synchronize cell cycle directly via the control of cellular cholesterol levels.

\subsection{Isoprenyl residues}

Two key intermediates of the mevalonate pathway, which influence a broad range of cellular processes are the two isoprenyls FPP and GGPP, which play a central role in protein prenylation. The latter is a process, where a farnesyl- or a geranylgeranyl moiety is transferred to the carboxyl terminus of a protein (Casey and Seabra, 1996; Maurer-Stroh et al., 2003; Roskoski, 2003; Zhang and Casey, 1996). Eukaryotes have three distinct enzymes responsible for prenylation, farnesyl transferase (FTase) and geranylgeranyl transferase (GGTase I and II). FTase and GGTase act on proteins, which contain a cysteine as the fourth residue from the carboxy terminal, in what is called a CAAX box; where $C$ represents the prenylated cysteine, $\mathrm{A}$ is an aliphatic amino acid, and $\mathrm{X}$ are amino acids, which determine whether the protein will be farnesylated or geranylgeranylated. GGTase II does not require a consensus sequence, but carries out a double prenylation on two terminal cysteins of Rab proteins (Maurer-Stroh and Eisenhaber, 2005).

The number and importance of substrate proteins, which undergo prenylation, highlights the significance of prenylation Several hundreds of proteins have the C-terminal motif required for prenylation, however, not all have yet been proven to be prenylated. Prenylated proteins include many signal transduction pathway proteins such as Ras, Rab, Rho and nuclear lamins (Sebti, 2005). Therefore, prenylation plays an important role in the physiological function of many pathways, and influences cell growth, differentiation and tumorigenesis. Protein prenylation results in the translocation of otherwise cytosolic proteins to the cell membrane via the hydrophobic prenyl anchor. Disruption of prenylation, either by a mutation of the cysteine or by blocking the mevalonate pathway, disrupts the membrane association. Furthermore, prenylation influences the function of many proteins, and thus lack of, or impairment of prenylation often results in dysfunctional proteins (Yang et al., 2000; Cox et al., 1992; Kato et al., 1992).

\section{Ras proteins}

Proteins that undergo prenylation are involved in a range of diseases where the prenylation plays a direct role in the progression of the disease. The Ras superfamily includes various small GTPbinding proteins classified in 6 families (Fig. 3). Ras proteins are involved in signal transduction pathways, implicated in regulation of cell growth, differentiation and cell survival. Many members of the Ras protein superfamily are polyprenylated, and in addition, some contain other posttranslational hydrophobic modifications such as palmitoylation, which further controls membrane association (Hancock et al., 1989; Boureux et al., 2007).

Ras proteins are expressed in a wide range of cells, where the proteins play a variety of different functions. Studies revealed that Ras proteins are not only associated with the plasma membrane, but also with intracellular membranes of various organelles, including endosomes, endoplasmic reticulum, Golgi apparatus and mitochondria (Choy et al., 1999). Under normal conditions, receptors such as receptor tyrosine kinases, $\mathrm{G}$-protein-coupled receptors and integrins, found in the membranes where Ras proteins reside, are activated by intra- and extracellular ligands and signaling molecules, and in turn activate Ras proteins by supplying GTP. Ras proteins function as GTP-GDP switches, and are confined in an inactive state when bound to GDP. They become active when supplied with GTP, which results in activation of further downstream targets (Scheffzek et al., 1997). By the GTPase function, the GTP is hydrolyzed and Ras returns to the inactive state until further GTP is supplied. 


\subsection{Ras subfamily}

The Ras subfamily is a group of small GTPases, which belong to the Ras-like proteins, and have given the family its name. There are three human Ras genes KRAS, HRAS and NRAS, which encode a total of four proteins due to alternative splicing of KRAS. Between 9 and $93 \%$ of tumors, depending on the specific type, and a total of up to $30 \%$ of all human cancers harbor a mutation in a Ras protein (Berndt et al., 2011; Bos, 1989). The Ras oncogenes are usually products of single amino acid mutations in one of the three genes, primarily KRAS, which renders the GTP switch of the protein dysfunctional, hence resulting in a constitutively active protein. Proper translocation of Ras proteins to the cell membrane is however, also crucial for oncogenic activity. Disruption of prenylation results in Ras proteins being present in the cytosol and being constitutively inactive (Jackson et al., 1990). Ras proteins can be either farnesylated or geranylgeranylated, and the addition of the appropriate prenyl is important for intact protein function. An exception are the Ras oncogenes, which retain the oncogenic activity regardless of the prenyl modification (Cox et al., 1992). Ras proteins are switched between active and inactive forms by hydrolyzing GTP into GDP. Therefore, they are also termed Ras-GTPases.

Ras-like proteins are involved in the control of many cellular processes, including cell proliferation, cell morphology and vesicle trafficking. The activation of Ras proteins results in activation of a large number of different signaling pathways, including the Raf-MEK-MAPK and the PI3K-AKT, both of which are involved in cell cycle progression, growth and survival. In addition to the GTPases themselves, the function of Ras proteins depends on at least three effector protein classes, which modulate the activity of the GTPase-switch. These include: (i) guanine nucleotide exchange factors (GEF), which increase activity by removing hydrolyzed GDP, (ii) GTPase-activating proteins (GAP), which decrease the activity by stimulating fast GTP hydrolysis, and (iii) GDP-disassociation inhibitors (GDI), which lock the Ras protein in the inactive, GDPbound form (Fig. 4) (Vega and Ridley, 2008; Sasaki and Takai, 1998).
Table 1

Rho family.

\begin{tabular}{ll}
\hline Subfamily & Modification \\
\hline Rac & Geranylgeranylation \\
RhoA & Geranylgeranylation, no isoprenylation \\
Rho B & Geranylgeranylation, farnesylation \\
Rho H & Geranylgeranylation, farnesylation \\
Rho F & Geranylgeranylation \\
Rho D & Farnesylation \\
Rho BTB & No isoprenylation \\
Rho T & No isoprenylation \\
Rnd & Farnesylation \\
Cdc 42 & Geranylgeranylation, farnesylation, no isoprenylation \\
\hline
\end{tabular}

The correct function of Ras proteins is highly dependent on membrane localization, and the function and effect of Ras depends on the cell type and membrane to which the protein is localized. Because membrane localization is key to the correct function of Ras-like proteins, prenylation is a very important process for their correct function (Li et al., 2004).

\subsection{Rho proteins}

Rho proteins are members of Ras-superfamily. Rho family consists of over 40 members, which can be subclassified into subfamilies (Table 1). The Rho family has some unique properties as its members can undergo either geranylgeranylation, farnesylation, both processes, and neither.

Rho GTPases were initially associated with the proper function of the cytoskeleton, mainly the organization of actin filaments, assembly of structural filaments and focal adhesions (Ridley and Hall, 1992; Paterson et al., 1990). This function is primarily mediated by Rho-associated protein kinases (ROCKs), which are activated by Rho. ROCK influences several effector proteins involved in the formation, depolymerization and directional

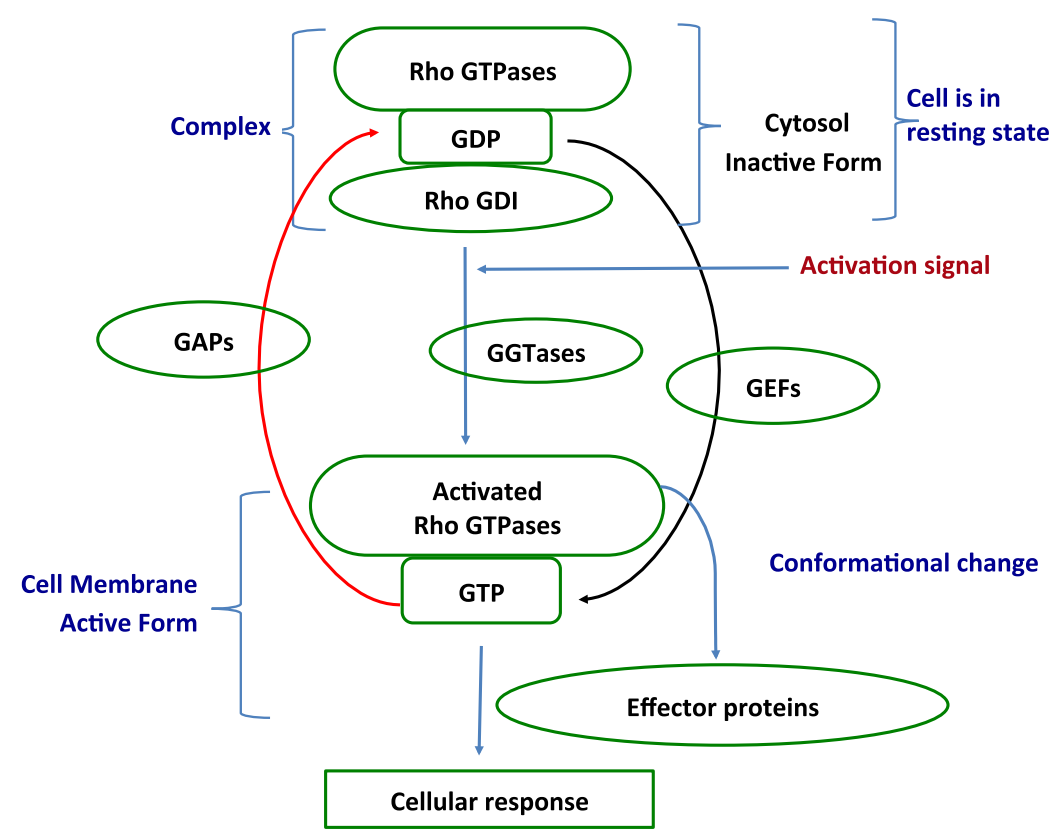

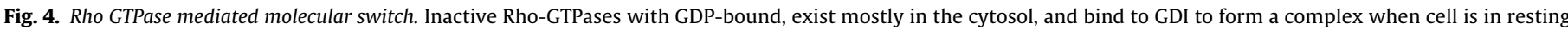

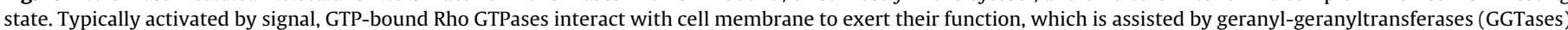

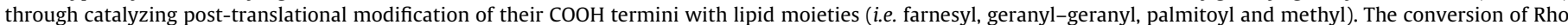

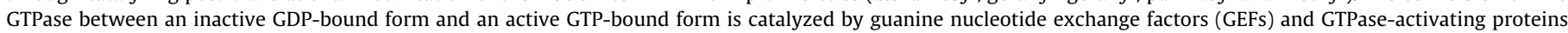
(GAPs). The active Rho proteins interact with effector proteins and initiate a downstream cellular response, allowed by the conformational change. 
growth of actin filaments, and therefore Rho plays a somewhat direct role in the control of cell movement and growth (Hall, 1998). Inhibition of Rho-GTPases Rho, Rac and Cdc42 control several steps in the M- and G1 phases of cell cycle, where reorganization of actin filaments is important. Rho GTPases also interact with a broad range of signal transduction pathways, and play a role in proliferation, gene transcription, cell cycle progression, vesicular transport and regulation of enzyme activity. Among others, Rho-GTPases activate the JNK-, MAP-kinase and NFKB pathways, however, their activation and the impact is highly dependent on the cell type (Zhang et al., 1995; Perona et al., 1997).

A group of Rho-specific GDIs (RhoGDI) exist which are structurally distinct from GDIs of other Ras proteins. In addition to the normal GDI function of locking the Rho in a GDP-bound state, the RhoGDIs have a large hydrophobic cavity, which is thought to interact with the prenyl. This interaction results in the covering of the prenyl, and therefore the reversible release of the Rho protein from the membrane, thereby disrupting the correct activation of the GTPase by membrane-bound receptors (Keep et al., 1997).

\section{Rho proteins and cancer}

In contrast to Ras oncogenes, which harbor activating mutations, Rho proteins are rarely mutated in human cancers. However, expression and activity levels of Rho-GTPases often vary between malignancies and healthy tissues. Due to the large number of effector proteins and signaling pathways involved, the role of RhoGTPases in malignancies depends on subcellular localization and function. High levels or activity of RhoA is generally linked to malignancy, and is associated with tumor progression and ability to metastasize (Kamai et al., 2004). RhoC is not essential for tumor progression, however when overexpressed, it highly increases the ability of tumor cells to undergo metastasis (Clark et al., 2000). Accordingly, knockdown or inhibition of RhoA and RhoC activity blocks the proliferation and invasiveness of tumor cells (Pille et al., 2005). Conversely, RhoB inhibits tumor proliferation and invasiveness, and is downregulated in many cancers. RhoB plays an important role in endosomal trafficking of signaling proteins and therefore lack of RhoB disrupts the correct response to external stimuli (Smith et al., 2004; Adini et al., 2003). Furthermore, RhoB is required for certain apoptotic responses of transformed cells, supporting the role of RhoB as a tumor-suppressor (Liu et al., 2001). RhoB expression is highly regulated, and stress, radiation, cytokines or toxins induce rapid increase of its expression levels (Huang and Prendergast, 2006).

Prenylation is crucial for the function of all Rho-GTPases, and adds an important dimension to the important roles that GTPases play in cell signaling and oncogenesis. RhoA is found exclusively in a geranylgeranylated or non-prenylated state, and prenylation can therefore be inhibited by the blockade of GGTaseI. In contrast, RhoB is primarily farnesylated (RhoB-F), but can also be geranylgeranylated (RhoB-GG), and the specific type of prenylation determines the subcellular localization of the protein. Inhibition of farnesylation results in a reduction of RhoB-F levels, and a subsequent gain of RhoB-GG, which results in changes in subcellular localization of RhoB. Studies suggest that there is a functional difference between RhoB-F and RhoB-GG, such that the anti-proliferative effect of RhoB increases when farnesylation is inhibited, and RhoB is primarily geranylgeranylated (Du et al., 1999). Although nonprenylated RhoB is generally considered inactive, it has been suggested that some activity is retained, including the activation of steroid-response element-dependent transcription (Lebowitz et al., 1997).

\section{Drugs influencing the mevalonate pathway}

\subsection{Statins}

Statins are a class of drugs originally discovered in fungi, however nowadays, many members are synthetic. Naturally occurring statins are found in various organisms such as oyster, mushroom, Aspergillus as well as Monascus fungi (Gunde-Cimerman and Cimerman, 1995; Liu et al., 2006). The statin group of drugs currently comprises of seven compounds: lovastatin, simvastatin pravastatin, fluvastatin, atorvastatin, rosuvastatin, and pitavastatin (Schaefer et al., 2004). In 2001, cerivastatin was withdrawn from the market due to an increased risk of myopathy including rhabdomyolysis. Statins were first approved for the treatment of hypercholesterolemia. However, due to their pleiotropic antiatherogenic properties (Fig. 4) they are also used in the secondary prevention of cardiovascular diseases. Their broader applications are under investigation, including Alzheimer's disease, osteoporosis and cancer treatment (Graaf et al., 2004a; Simons et al., 2001; Jick et al., 2000; Wolozin et al., 2000; Aggeler et al., 2002).

Statins competitively inhibit HMGR by blocking the conversion of HMG-CoA to mevalonate (Holdgate et al., 2003). The molecular mechanism underlying inhibition of HMGR by statins is a catalytic mechanism. The statins occupy the catalytic site of HMGR, specifically the binding site of HMG-CoA by numerous polar and van der Waals interactions, thus blocking access of this substrate to the active site (Istvan, 2003; Istvan, 2002; Buhaescu and Izzedine, 2007). Rosuvastatin is the competitor with the largest affinity for HMGR in the statin group (Holdgate et al., 2003).

Inhibition of HMGR which is the primary drug target of statins administered for cardiovascular disease treatment and prevention, results in disruption of de novo cholesterol biosynthesis, primarily in the liver. This results in activation of the production of microsomal HMGR and cell surface LDL-receptors on hepatocytes (Lennernas and Fager, 1997). This consequently leads to circulatory LDL being taken up into cells where they are metabolized and converted into the bile salts, resulting in significant reduction of LDL levels in the blood (Ma et al., 1986).

Apart from the major role in lowering cholesterol levels, statins are believed to play a significant role in inhibition of tumor growth and differentiation (Graaf et al., 2004a,b; Denoyelle et al., 2001). However, this application brings a great deal of controversy among oncologists (Graaf et al., 2004b; Shepherd, 2006). Statins induce cell growth arrest, apoptosis, inhibit metastasis, and angiogenesis. The mechanisms underlying these effects are not fully understood. The latest findings indicate that Ras farnesylation is only one of processes causing statins to inhibit cancer. For instance, cerivastatin inhibits both proliferation and invasion of aggressive breast cancer cell lines via inactivation of RhoA by inhibition of geranylgeranylation (Denoyelle et al., 2001). In vivo animal experiments show that HMGR activity correlates with neuroblastoma tumor growth in mice (Maltese et al., 1985), where treatment with statin drugs blocks tumor progression in mice but also development of colorectal cancer in rats by inhibition of isoprenylation of rasp21 (Graaf et al., 2004a; Iishi et al., 2000). In effective cancer treatment, it is suggested that statins act most effectively together with chemotherapeutics in combination cancer therapy. Recent studies reveal beneficial antineoplastic properties of statins, by altering apoptosis inhibitory effect of VEGF and limiting secretion of matrix metalloproteinases (Denoyelle et al., 2001; Jaswal et al., 2003).

\subsection{Bisphosphonates}

Bisphosphonates (BPs) are sub-divided into two classes, distinguished by their chemical structures and the mode of action (van Beek et al., 2003; Fleisch, 1998; Papapoulos, 2008). The first 
group, devoid of nitrogen in their backbone, consists of etidronate, clodronate and tiludronate, whereas the second group containing nitrogen, consists of alendronate, ibandronate, olpadronate, pamidronate, risendronate and zolendronate (van Beek et al., 2003). BPs play an essential role in bone disease treatment, including osteoporosis, Paget's disease, decreasing excessive bone resorption (Kotwal et al., 2013; Fleisch, 1991). Furthermore, BPs have pro-apoptotic potential by inducing osteoclast apoptosis, which has led to their application in skeletal associated disorders (Papapoulos, 2008; Zeman and Shipman, 1997). Nitrogenous BPs are pivotal in targeting FPP-synthase, a key enzyme in the mevalonate pathway, resulting in decrease in the biosynthesis of FPP and GGPP. Inhibition of FPP synthase further leads to the accumulation of IPP, which is converted to the ATP analogue ApppI. ApppI is cytotoxic, and leads to the direct activation of mitochondrial apoptosis, which can lead to undesired side effects (Monkkonen et al., 2006). Therefore, these features of BPs can potentially restrict malignant cell transformation or tumor progression.

Nitrogen-containing BPs display anti-proliferative and antitumor potential, also resulting from mevalonate pathway inhibition. Inhibition of prenylation of small GTPases causes lack of integrinmediated tumor cell adhesion to the bone (Coxon et al., 2004), accumulation of unprenylated Rap1 and therefore growth inhibition of mesothelioma cells (Wakchoure et al., 2006). Inhibition of geranylgeranylation results in Rho protein deactivation, hence interfering with cancer cell migration (Denoyelle et al., 2003).

\subsection{Farnesyl-transferase inhibitors}

Farnesyl-transferase inhibitors (FTIs) represent a new generation of signal transduction inhibitors, developed to target the critical post-translational modification of Ras (Buhaescu and Izzedine, 2007). FTIs include compounds competitive with either farnesyl pyrophosphate, competitive with the CAAX domain in FTs or compounds with both activities (Crul et al., 2001). The activity of any of these molecules results in inhibition of Ras farnesylation and cell growth arrest (Appels et al., 2005a). FTIs nonspecifically inhibit farnesylation, and therefore disrupt functions of many different cellular proteins, and thus can act in a Ras-independent manner (Buhaescu and Izzedine, 2007; Appels et al., 2005a; Basso et al., 2006). Inhibition of RhoB farnesylation is, for example, unlikely to reduce the amount of prenylated RhoB, since, as previously described, RhoB can also be geranylgeranylated. However, since RhoB-GG has a potential anti-proliferative effect, this might further enhance the potency of FTIs (Berndt et al., 2011).

It has been suggested that FTIs can potentially be applied in cancer treatment. Lonafarnib was the first FTI, which has entered a clinical trial, followed by several others. For most cases clinical trials in phase I and II were successful displaying a substantial antitumor activity with reasonable toxicity profile, but none of FTIs passed phase III trial showing no significant effects on survival in solid cancers (Appels et al., 2005a; Brunner et al., 2003; Appels et al., 2005b).

Some studies revealed that certain Ras proteins, when farnesylation is inhibited, can become active by alternative geranylgeranylation conducted by GGTase-I, which is confirmed in a $\mathrm{K}$-Ras-dependent mouse model, showing that farnesylation is not crucial for tumorigenesis (Whyte et al., 1997; Mijimolle et al., 2005).

\subsection{Geranyl transferase inhibitors}

A novel class of drugs targeting the GGTase, geranylgeranyl transferase inhibitors (GGTIs), has recently been developed. GGTI development has utilized several different strategies including peptidomimetic inhibitors and small-molecule inhibitors, with promising anti-tumor properties (Sane et al., 2010; Peterson et al.,
2006). GGTase-I-deficient cells accumulated $\mathrm{p} 21^{\mathrm{CIP} 1 / \text { Waf } 1}$ and failed to proliferate (Vogt et al., 1997). Similarly as for farnesylation, some of the GGTase substrates can undergo alternative prenylation or farnesylation, when GGTase inhibition occurs, and preserve the basic activity (Philips and Cox, 2007). GGTase-I deficiency in mice ameliorates the growth of K-Ras-driven lung tumors, however it is not required for the development of myeloid cells (Sjogren et al., 2007).

When administered simultaneously, GGTIs and FTIs have a synergistic inhibitory effect on K-Ras prenylation, resulting in even greater apoptotic response in contrast to each of the inhibitors working separately in vitro (Lobell et al., 2001). The combination of GGTI-2Z with lovastatin, results in $\mathrm{G}_{0} / \mathrm{G}_{1}$ phase arrest in STS26 T-cells and their co-administration could potentially reveal new therapeutic applications for mevalonate pathway inhibiting drugs (Sane et al., 2010).

\section{Regulation of stemness by Rho GTPases activated by isoprenoids}

\subsection{Stem cell and cancer stem cells}

Stem cells proliferate indefinitely while maintaining pluripotency. According to their origin, stem cells could be divided, for example, into embryonic, fetal, umbilical-, adult-, and inducedpluripotent stem cells (iPS). Reya et al. (2001) postulated that a striking similarity could be found between stem cells and some CSC. CSC can be defined as a small subgroup of cancer cells with indefinite potential for self-renewal that drives tumorigenesis. CSC constitute a small subpopulation of cancer cells within a tumor. They can differentiate into heterogeneous lineages of cancer cells. CSC within a tumor are believed to be responsible for the re-growth of the tumor. For the cancer to be completely eradicated, all cancer cells including CSC need to be eliminated. They are also believed to be responsible for the occurrence of metastasis (Wasik et al., 2014). The difference between normal stem cells and CSC is their dependence on a special microenvironment in which they reside; so called stem cell niche (Wasik et al., 2014; Farahani et al., 2014). The latter is important in maintaining stemness and preventing tumorigenesis by providing inhibitory signals for both proliferation and for regulation of differentiation. When required, the niche provides transient signals for cell division to support ongoing tissue regeneration. This balance between signals promoting and inhibiting cell proliferation is necessary for maintenance of stem cells function. CSC may arise as a result of intrinsic mutations leading to self-sufficient cell proliferation which may include deregulation of the balance of niche's signals (Farahani et al., 2014). Elements of the embryonic signaling cascades such as Notch, Sonic hedgehog, Wnt- $\beta$, are active in both normal stem cells and CSC (Wasik et al., 2014).

The stem cell niche is vital for the maintenance of "stemness" and also in the prevention of tumorigenesis by the provision of inhibitory signals for proliferation and differentiation (Farahani et al., 2014). The Rho family of small GTPases plays a crucial role in stem cell proliferation. As already outlined earlier, a series of post-translational modifications are essential for Rho GTPases to be functionally active. Prenylation of Rho proteins is crucial for the insertion of active Rho proteins into the membranes, where they bind to their respective effectors and are involved in a variety of cellular events including cell proliferation and differentiation, the two main characteristic features of stem cells. Thus, Rho GTPases and in turn the isoprenoids which activate GTPases are involved in regulating the "stemness" of these cells.

Yoon et al. demonstrated that the Rho family GTPase Rac1 is involved in glioma stem-like cell (GSLC) maintenance in human glioma. Knock-down of Rac1 resulted in a substantial reduction in 


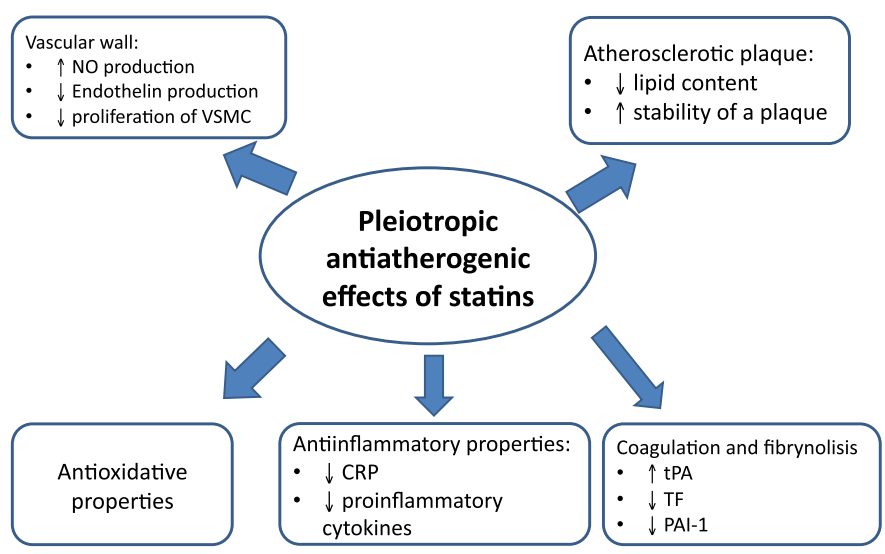

Fig. 5. Pleiotropic anti-atherogenic properties of statins. Statins exert beneficial antiatherogenic effects through several mechanisms. They decrease lipid-levels in circulation, while stabilizing atherosclerotic plaque. Statins exert anti-inflammatory and antioxidant properties, furthermore, they foster fibrinolysis while counteracting coagulation. They also exert various positive effects on the vascular wall, they promote vasodilatation by stimulation of NO-production, and they decrease endothelin expression and proliferation of vascular-smooth muscle cells (VSMSC).

the expression of GSLC markers. The activation of Rac1 is also essential for the self-renewal of GSLC indicating that the small GTPase Rac1 regulates stemness in GSLCs (Yoon et al., 2011).

Statins inhibit cell proliferation and lead to loss of self-renewal capacity in mouse embryonic stem cells (ESCs) (Lee et al., 2007). Lee et al. has shown that down-regulation of ESC self-renewal by simvastatin is significantly associated with its inhibitory effect on RhoA geranylgeranylation. This group demonstrated that the basal RhoA activity is required to retain self-renewal capacity and the undifferentiated state of ESCs, the loss of which might initiate the differentiation program in ESCs. The simvastatin-induced down-regulated expression of the alkaline phosphatase ESC marker clearly points at the role of isoprenoids in the retention of the selfrenewal capacity of stem cells.

Statins inhibit HMGR which catalyzes the conversion of HMG$\mathrm{CoA}$ to mevalonate. In turn, inhibition of mevalonate biosynthesis blocks the biosynthesis of downstream isoprenoid intermediates in the pathway like FPP and GGPP which are isoprenoid substrates for protein farnesylation and geranylgeranylation, respectively (Zhang and Casey, 1996). HMGR inhibitors also inhibit cell proliferation by prevention of transition from $G_{1}$ to $S$ phase in the cell cycle, low expression of cyclin-dependent kinases facilitating cell cycle progression or upregulation of cell cycle inhibitors, the inhibition of cell proliferation being reversed by free mevalonate, FPP, or GGPP; this emphasizes the role of mevalonate and its downstream isoprenoid products in the maintenance of cell proliferation (Gauthaman et al., 2009).

Rho signaling is also essential for actin-cytoskeletal remodeling (Fig. 5) and the expression of cell adhesion molecules (CAMs) (Ellenbroek and Collard, 2007). ROCK, a kinase associated with RhoA phosphorylates various downstream targets of ROCKs. Direct inhibition of ROCK has adverse effects on morphological changes and down-regulates ESC markers. The actin cytoskeleton is generally monitored by many of the downstream targets of ROCKs, like myosin-binding subunit that is present on the myosin light chain phosphatase, myosin light chains. Furthermore, through their interaction with media [FH (formin homology) domain containing protein] Rho proteins enhance actin polymerization into linear filaments. Rac and Cdc42 are also believed to be involved in actin polymerization. Once Rac releases the WAVE [WASP (Wiskott-Aldrich syndrome protein) verprolin homologous] from an inhibited complex, the WAVE protein activates the heptameric actin polymerizing complex Arp2/3 (actin-related protein 2/3). In

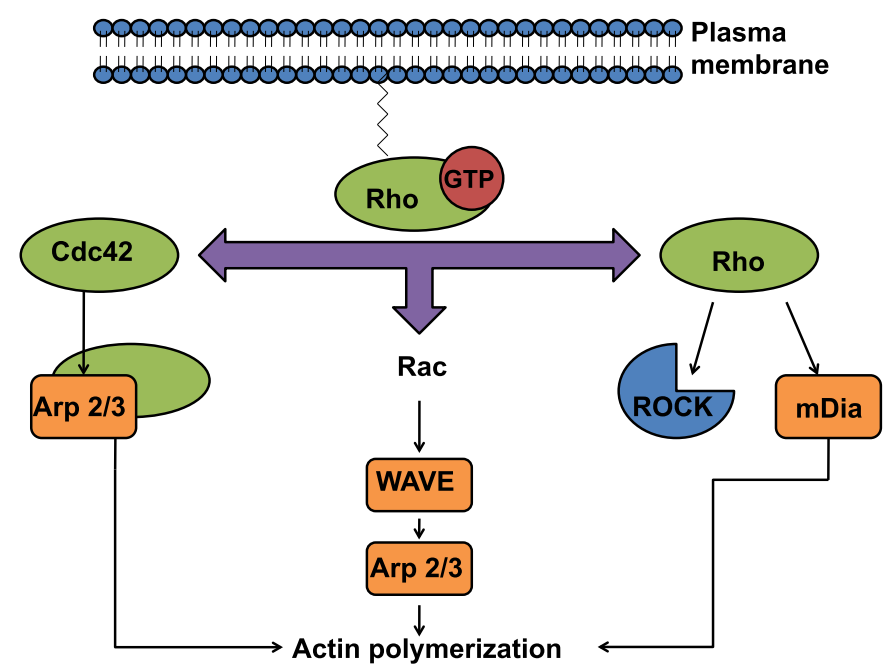

Fig. 6. Rho GTPase mediated actin polymerization. The signaling cascade activated by the isoprenylated Rho proteins is necessary for cell proliferation and thus also in the maintenance of "stemness". The downstream targets of active Rho GTPases, with bound GTP, include kinases like ROCK, nucleation promoting factors including mammalian Diaphanous formin, mDia; Wiskott-Aldrich syndrome protein, WASP Wiskott-Aldrich syndrome protein-family verprolin homologous protein, WAVE. mDia produces unbranched actin filaments while WASP and WAVE interact with Arp2/3 complex and generate branched microfilaments. Rho GTPase also promotes myosin actin interactions through ROCK, which phosphorylates actin cytoskeleton regulators. Cdc42 directly activates Arp2/3 complex.

turn, activated Arp2/3 helps in the elongation of actin filaments from the sides of already existing filaments, which creates a meshwork. Cdc42 activates Arp2/3 by directly interacting with another Arp2/3 activator, neuronal-WASP (Fig. 6).

RhoA, Rac1 and Cdc42 affect cell cycle progression and growth as well as apoptosis, by regulating transcription of specific genes, including cyclin D1. The latter is involved in the stimulation of the transition of $G_{1}$ to $S$. The transcription of cyclin D1 is controlled by transcription factors like ETS, AP- 1 and NFKB, the activity of which is regulated by Rho GTPases including RhoA, Rac1 and Cdc42. RhoA signaling also modulates the levels of the Cyclin Dependent Kinase Inhibitors (CDKIs) p2 $1^{\text {WAF1 }}$ and p27 $7^{\text {KIP1 }}$ (Sahai and Marshall, 2002).

\section{Regulation of stemness by isoprenoids with focus on small Rho GTPase-dependent pathways}

Small GTP-binding proteins of the Ras superfamily modulate diverse cellular functions such as cytokinesis, cell motility, cell adhesion and cell proliferation. These proteins are the upstream elements of various signaling pathways. Many of the Rho family GTPases are involved in uncontrolled proliferation, cell survival, loss of epithelial cell properties, abnormal cell migration, and metastasis (Yoon et al., 2011).

Inhibition of Rho proteins by statins results in blockade of growth and proliferation of neoplastic cells. Hence, the role of isoprenoids in the regulation of stemness has been in the focus of recent research. Lee et al. (2007) evaluated the influence of simvastatin on mouse ESCs. Use of this statin resulted in the loss of self-renewal of mouse ESCs determined by a marked downregulation of ESC-markers: alkaline phosphatase, Oct4, Nanong, Rex- 1 and SSEA-1. These effects were brought about by the depletion of the intracellular pool of GGPP but not FPP. GGPP is critical for ESCs self-renewal. Inhibition of geranylgeranylation of small GTP-binding proteins resulted in decreased levels of ROCK2 protein in ESCs. Pluripotent ESCs have a round colony shape that is tightly packed and close together. This tight intercellular contact is diminished by simvastatin. The morphological changes were 
accompanied by a reduced amount of membrane-bound cell adhesion molecules: PECAM-1, VCAM-1 and ICAM-1. These effects were reversed by supplementation of GGPP indicating that Rho/Rhokinase signaling was required for actin-cytoskeletal remodeling and expression of membrane-bound cell adhesion molecules.

Rac1-dependent signal transduction is essential for malignant cell transformation. Deregulated expression and activation of Rac1 is involved in the development and progression of cancer. High expression of this protein correlates with rapid proliferation and survival of glioma cells. Yoon et al. (2011) observed that in GSLC, the Rac1-Pak signaling axis was markedly activated and Rac1 knockdown caused a reduction in expression of GSLC markers. These results were detected with no change in Rac1 protein levels. The inhibition of Rac1 expression with siRNA resulted in the inhibition of self-renewal potential measured as sphere-forming ability of glioma cells. Downregulation of Rac1 in monolayer-cultured glioma cells did not suppress cell growth suggesting that Rac1 was required only for the proliferation of glioma stem-like cells but not monolayer cultured cells. Inhibition of Rac1 with siRNA in glioma U87 and U373 cell lines resulted in a decrease of selfrenewal related protein levels of Sox2, Notch2, and $\beta$-catenin in these cell lines (Yoon et al., 2011). Migration, invasion and malignant transformation were also attenuated. The results of this study indicate that activation of Rac1 is involved in the enrichment of the GSLC subpopulation in glioma and the maintenance of malignant tumors.

Receptor for hyaluronan (HA)-mediated motility (RHAMM) also known as HMMR, IHABP or CD168 is a breast cancer susceptibility gene with dual oncogenic functions as an HA receptor and mitotic spindle binding protein. Generally, RHAMM is not detected under physiological conditions. Its transient expression is observed during wound healing, but it is overexpressed in neoplastic tissues. RHAMM binds to mitotic spindle and promotes interphase microtubule instability and mitotic spindle integrity. It is also exported to the extracellular surface to partner with CD44, enhancing CD44-mediated tumor progression via ERK1/2-association and maintaining high proliferative activities and motility of invasive cancer cells (Hamilton et al., 2007). The Hippo-YAP pathway with the transcriptional coactivator Yes-associated protein (YAP) is critical in regulating organ size, tissue regeneration and stem cell renewal. The Hippo-YAP pathway is involved in tumorigenesis and tumor progression. The Hippo-YAP pathway consists of kinase cascade in which Mst1 or Mst2 forms complex with the adaptor protein WW45 and phosphorylates the LATS1/2 kinases and adaptor protein MOB. The LAST/MOB complex subsequently phosphorylates YAP at Ser127. Other components of the Hippo-YAP pathway include NF2, WW45 and KIBRA. Upregulation of YAP and its nuclear localization strongly correlates with poor prognosis and tumor progression in many cancers (Piccolo et al., 2014). YAP can promote metastasis through interaction with the TAED/TEF transcription factors.

The mevalonate pathway promotes the expression of RHAMM via transcriptional mechanisms mediated directly by YAP leading to ERK-phosphorylation. These processes are necessary for breast cancer cell migration and invasion. RHAMM is a unique transcriptional target gene of YAP-TAED of the Hippo pathway. The mevalonate pathway modulates YAP phosphorylation via Rho GTPase activation and actin cytoskeleton rearrangement independent of MST and LATS kinase activity. The core kinases of Hippo-YAP signaling MST1/2 and LATS1/2 control the phosphorylation level and activity of YAP. The Hippo pathway plays a key role in organ size control and tissue regeneration. It is also necessary for stem cell differentiation and control of proliferation. Deregulation of the Hippo-YAP pathway is observed in many human carcinomas including lung, colorectal, ovarian and liver cancer. YAP/TAZ share relationships with morphogenic signals such as Wnt-dependent growth factors, and are regulated by Rho and GCPR (Wang et al., 2014; Piccolo et al., 2013). YAP/TAZ promotes cell proliferation and inhibits differentiation, particularly in stem cells and organ-specific progenitors (Ramos and Camargo, 2012). EMT is a dynamic and reversible cellular event in which cells lose epithelial features, such as polarity and intercellular junctions, and acquire mesenchymal characteristics, leading to increased migration and invasion (Kalluri and Weinberg, 2009). Silencing of TAZ in glioma CSC decreased expression of mesenchymal markers, invasion, self-renewal, and tumor formation (Bhat et al., 2011).

In various cancers, the Hippo-YAP pathway is often perturbed through cross-talk with a number of key signaling pathways which frequently harbor oncogenic mutations within Wnt, TGF- $\beta$, BMP, GRCP, Hedgehog, insulin, mTOR and Notch (Harvey et al., 2013). The Rho protein family is closely linked to cancer as their deregulated activation drives transformation. The elevated expression of these proteins is associated with several human neoplasia. RhoA and RhoC are thought to have a role in metastasis. The Hippo-YAP pathway is regulated by GPCR signaling. Cell surface GPCR is coupled to RhoA and subsequent GTPase activation leads to LATS1/2 inhibition and induction of YAP and its paralogue TAZ, and RhoA activation induced actin cytoskeleton reorganization, which plays an important role in Hippo-YAP signaling.

YAP and TAZ are the mediators of the Hippo-YAP pathway, hence, the mevalonate pathway controls YAP/TAZ activity (Sorrentino et al., 2014). In tumor cells, sterol regulatory element binding protein (SREBP) is induced by its oncogenic cofactor, mutant p53. Increased transcriptional activity of SREBP leads to an augmented activity of HMGR, which is the rate-limiting enzyme in the mevalonate pathway. The product of this enzyme, mevalonate, is a substrate for the biosynthesis of GGPP. Rho GTPase is activated by posttranslational modification, geranylgeranylation. The greater is the production of GGPP, the higher is the activity of Rho GTPase, which, in turn, activates YAP/TAZ by inhibiting their phosphorylation and promoting their nuclear accumulation. Aberrant YAP/TAZ activation is frequently observed in a variety of human cancers.

The Cadherin-11 gene (CDH11, OB-cadherin) is broadly expressed in human normal adult and fetal tissues. This gene is often silenced and methylated in all tumor cell lines but not in immortalized epithelial cells. CDH11 inhibits Wnt/ $\beta$-catenin and AKT/Rho signaling. CDH11 functions as a pro-apoptotic tumor suppressor through antagonizing $W n t / \beta$-catenin. It also inhibits stemness of tumor cells and their migration and invasion (Li et al., 2012). The $W n t / \beta$-catenin signaling is essential for stem cell regulation and tumorigenesis. Overexpression of $\beta$-catenin increases proliferation of head and neck squamous cell carcinoma (HNSCC) and induces dedifferentiation of these cells to cells with stem-cell like features. $\beta$-catenin also directly regulates Oct4 transcription in HNSCC stem-like cells (Lee et al., 2014). Oct4 is an important regulator of embryonic stem cell fate. Koo et al. (2014) observed that Oct4 regulates stemness of HNSCC and causes a dedifferentiation of differentiated HNSCC to CSC.

Asad et al. (2014) observed that Stem-A type frizzled family receptor 7 (FZD7) which serves as Wnt-receptor, was overexpressed in a subgroup of ovarian cancer cells. This pathway could be broadly divided into canonical and non-canonical (Veeman et al., 2003). In the non-canonical Wnt/planar cell polarity (PCP) pathway, the Wnt ligand stimulates the activities of several small GTP-binding proteins including RhoA, Rac1 and cdc42, which regulate diverse processes such as cytoskeleton rearrangement, cell migration and polarity. Alterations in this Wnt/PCP pathway trigger the initiation and progression of malignant phenotype through the stimulatory effect on cell proliferation, survival, abnormal tissue polarity, cell invasion and cancer metastases (Katoh, 2005; Ridley, 2004). Among 10 known FZD receptors, FZD7 mediates both canonical and non-canonical Wnt-signaling. FZD7 is thought to regulate 
the pluripotency and self-renewal of Stem-A subtype of ovarian cancer (Melchior et al., 2008; Fernandez et al., 2014). FZD7 is ubiquitously expressed in pluripotent stem cells and has a role in cell fate determination. In an in vitro colorectal cancer morphogenesis culture system EMT of monolayer (mesenchymal-state) into 3D spheres (epithelial state) required FZD7 (Vincan et al., 2007). This receptor may be also required for this transition in ovarian cancer cells. A plausible mechanism of how FZD7 regulates the mesenchymal state is via the activity of small GTPases. Knockdown of FZD7 suppresses RhoA expression but upregulates Rac1 (Asad et al., 2014). The activation of RhoA is an important downstream effect in the non-canonical Wnt/PCP pathway to regulate cell motility, cell migration and invasion.

\section{Small Rho GTPases-independent effects of statins in cancer}

As the tumor grows, cancer cells promote angiogenesis. Cholesterol, one of the key end products of the mevalonate pathway, plays an indispensable role in blood vessel formation. It has been suggested that statins exert anti-angiogenic effects to prevent metastasis by down-regulating the pro-angiogenic vascular endothelial growth factor (VEGF), inhibition of endothelial cell proliferation, and blocking cell adhesion to the extracellular matrix (Frick et al., 2003; Weis et al., 2002). Lovastatin and simvastatin influence the expression of various proteins regulating cell cycle (p21 and p27) and have the ability to arrest cancer cells in the $G_{1} / S$ phases (Koyuturk et al., 2004; Rao et al., 1998). Statins may affect melanoma immunotherapy as statins can favor the development of specific adaptive immune responses and innate immune responses against human metastatic melanoma cells. Independently of Ras and Rho GTPases signaling pathways, statins can induce a weak but significant increase in membrane expression of MHC class I chainrelated protein $A$ in human melanoma cell lines treated with statins while this overexpression enhances the sensitivity of melanoma cells to NK cell-dependent lysis in vitro (Pich et al., 2013).

\section{Concluding remarks}

The mevalonate pathway attracted a significant attention in the past two decades after the successful Nobel Prize-winning efforts of two leader scientists in cholesterol metabolism cascade, Drs. Goldstein and Brown. As discussed earlier, mevalonate is the central engine for cholesterol production and is also responsible for precursor of prenylated proteins including FPP and GGPP. Statins are competitive inhibitors of rate determining step of this pathway (HMGCoA-reductase). Since their discovery, the statin superfamily of inhibitors has made significant impact on human health, and currently since statins patent protections has ended, or will end soon, several new applications of these compounds have been proposed in different diseases, including cancer, and fibrotic lung diseases. Although attempts to use statins in cancer therapy are not new, several pharmaceutical companies are involved in clinical evaluation of these inhibitors.

The function of the Ras proteins is extremely complex, with many aspects yet to be discovered. As our current understanding is increasing, it is becoming evident that prenylation is crucial for the correct function of Ras proteins, and also the role of Ras in disease. The mevalonate pathway is, therefore, becoming an increasingly attractive therapeutic target for a range of diseases, primarily focusing on cancer. By controlling the mevalonate pathway, it is possible to alter prenylation patterns, thereby influencing the activity of Ras proteins. Due to the very diverse roles of the proteins, and the differences between cell types and tissues, the therapeutic effects based on inhibition of the mevalonate pathway are varying, and the approach is not applicable as a general anticancer strategy, as only some tumor types are susceptible to this treatment (Fritz, 2009; Swanson and Hohl, 2006). A better therapeutic strategy could be achieved by targeting the mevalonate pathway in combination with other standard, or experimental treatments, like apoptins (Chaabane et al., 2014; Rollano Penaloza et al., 2014), or drugs preferentially targeting CSC, like salinomycin (Jangamreddy et al., 2013). Beside Ras and Rho, the function of several other proteins could be dramatically affected by subcellular localization. For example, nuclear-localized Akt may favor stemness-state of cells, but render them susceptible to apoptotic stimuli (Jain et al., 2015; Maddika et al., 2009; Magnusson et al., 2015).

Other possible strategies that one could consider are the application of statins, or prenylation inhibitors, as sensitizers in standard antitumor therapies. For example, in a recent study, involving 1562 patients diagnosed with glioblastoma multiforme during 2000-2009, Cox proportional hazard models have been used to compute hazard ratios (HRs) and 95\% confidence intervals (CI) for all-cause death associated with pre-diagnostic statin (most of the patients used simvastatin) use in these patients. Their results showed that pre-diagnostic statin treatment was associated with a reduced HR of death $(0.79 ; 95 \% \mathrm{CI}: 0.63-1.00)$ in the studied cohort (Gaist et al., 2014). As simvastatin (a lipophilic statin) passes the blood-brain-barrier, one should consider phase-I clinical trial study to test the combination of simvastatin together with the routine glioblastoma therapy-agent temozolomide. Hence, it would be interesting to test the effectiveness of statins, when combined with other glioblastoma-therapy approaches, as no effective treatment currently exists.

Statins or prenylation inhibitors could also be tested in the therapy of NSCLC. It has been reported that small RhoGTPase play an important role in small cell lung cancer (Bhattacharya et al., 2015; Naidoo and Drilon, 2016). Therefore, possible use of statins or prenylation inhibitors in combination with standard therapy of non-small cell lung cancer may improve the clinical outcome of this incurable and devastating disease. In such case the administration of statins or prenylation inhibitors could also be done with the use of an inhaler.

When designing and testing new therapeutic indications for statins, one has to consider the non-selective action of most statins and prenlyation inhibitors. The development of preferential delivery methods, targeting CSC would greatly simplify clinical applications of statins or prenylation inhibitors for cancer therapy.

In conclusion, while being a very promising field, the manipulation of the mevalonate pathway presents many difficulties. Due to the importance of the pathway, the number of therapeutic targets is very large, which could be seen both as a drawback, and as an opportunity. Most current drug candidates target the broad function of the pathway, which results in a large number of cellular processes being influenced. More detailed knowledge about the pathway, as well as downstream-effector functions, will open new avenues for more targeted therapeutic interventions.

\section{Acknowledgments}

ACP thankfully acknowledges the support from BK/265/RAU1/ 2014/t.10, and from GeCONiI (POIG.02.03.01-24-099/13). SG thankfully acknowledges the support from Manitoba Medical Service Foundation.

\section{References}

Adini, I., Rabinovitz, I., Sun, J.F., Prendergast, G.C., Benjamin, L.E., 2003. RhoB controls Akt trafficking and stage-specific survival of endothelial cells during vascular development. Genes Dev. 17 (21), 2721-2732.

Aggeler, R., Coons, J., Taylor, S.W., Ghosh, S.S., Garcia, J.J., Capaldi, R.A., et al., 2002. A functionally active human F1F0 ATPase can be purified by immunocapture from 
heart tissue and fibroblast cell lines. Subunit structure and activity studies. J. Biol. Chem. 277 (37), 33906-33912.

Appels, N.M.G.M., Beijnen, J.H., Schellens, J.H.M., 2005a. Development of farnesyl transferase inhibitors: a review. Oncologist 10 (8), 565-578.

Appels, N.M.G.M., van Maanen, M.J., Rosing, H., Schellens, J.H.M., Beijnen, J.H., 2005b. Quantitative analysis of the farnesyl transferase inhibitor lonafarnib (Sarasar(TM), SCH66336) in human plasma using high-performance liquid chromatography coupled with tandem mass spectrometry. Rapid Commun. Mass Spectrom. 19 (15), 2187-2192.

Asad, M., Wong, M.K., Tan, T.Z., Choolani, M., Low, J., Mori, S., et al., 2014. FZD7 drives in vitro aggressiveness in Stem-A subtype of ovarian cancer via regulation of non-canonical Wnt/PCP pathway. Cell Death Dis. 5, e1346.

Assaraf, Y.G., 2007. Molecular basis of antifolate resistance. Cancer Metastasis Rev. 26 (1), 153-181.

Basso, A.D., Kirschmeier, P., Bishop, W.R., 2006. Farnesyl transferase inhibitors. J. Lipid Res. 47 (1), 15-31.

Berndt, N., Hamilton, A.D., Sebti, S.M., 2011. Targeting protein prenylation for cancer therapy. Nat. Rev. Cancer 11 (11), 775-791.

Bhat, K.P., Salazar, K.L., Balasubramaniyan, V., Wani, K., Heathcock, L., Hollingsworth, F., et al., 2011. The transcriptional coactivator TAZ regulates mesenchymal differentiation in malignant glioma. Genes Dev. 25 (24), 2594-2609.

Bhattacharya, S., Socinski, M.A., Burns, T.F., 2015. KRAS mutant lung cancer: progress thus far on an elusive therapeutic target. Clin. Transl. Med. 4 (1), 35.

Bonetti, P.O., Lerman, L.O., Napoli, C., Lerman, A., 2003. Statin effects beyond lipid lowering-are they clinically relevant? Eur. Heart J. 24 (3), 225-248.

Bos, J.L., 1989. Ras oncogenes in human cancer: a review. Cancer Res. 49 (17) 4682-4689.

Boureux, A., Vignal, E., Faure, S., Fort, P., 2007. Evolution of the Rho family of Ras-like GTPases in eukaryotes. Mol. Biol. Evol. 24 (1), 203-216.

Brown, M.S., Goldstein, J.L., 1974. Suppression of 3-hydroxy-3-methylglutaryl coenzyme A reductase activity and inhibition of growth of human fibroblasts by 7-ketocholesterol. J. Biol. Chem. 249 (22), 7306-7314

Brown, M.S., Goldstein, J.L., 1980. Multivalent feedback-regulation of Hmg Coa Reductase, a control mechanism coordinating isoprenoid synthesis and cellgrowth. J. Lipid Res. 21 (5), 505-517.

Brunner, T.B., Hahn, S.M., Gupta, A.K., Muschel, R.J., McKenna, W.G., Bernhard, E.J. 2003. Farnesyltransferase inhibitors: an overview of the results of preclinical and clinical investigations. Cancer Res. 63 (18), 5656-5668.

Buhaescu, I., Izzedine, H., 2007. Mevalonate pathway: a review of clinical and therapeutical implications. Clin. Biochem. 40 (9-10), 575-584.

Burg, J.S., Espenshade, P.J., 2011. Regulation of HMG-CoA reductase in mammals and yeast. Prog. Lipid Res. 50 (4), 403-410.

Casey, P.J., Seabra, M.C., 1996. Protein prenyltransferases. J. Biol. Chem. 271 (10), 5289-5292.

Chaabane, W., Cieslar-Pobuda, A., El-Gazzah, M., Jain, M.V., Rzeszowska-Wolny, J., Rafat, M., et al., 2014. Human-gyrovirus-Apoptin triggers mitochondrial death pathway-Nur77 is required for apoptosis triggering. Neoplasia (New York, NY) 16 (9), 679-693.

Choy, E., Chiu, V.K., Silletti, J., Feoktistov, M., Morimoto, T., Michaelson, D., et al., 1999. Endomembrane trafficking of Ras: the CAAX motif targets proteins to the ER and Golgi. Cell 98 (1), 69-80.

Clark, E.A., Golub, T.R., Lander, E.S., Hynes, R.O., 2000. Genomic analysis of metastasis reveals an essential role for RhoC. Nature 406 (6795), 532-535.

Cox, A.D., Hisaka, M.M., Buss, J.E., Der, C.J., 1992. Specific isoprenoid modification is required for function of normal, but not oncogenic, Ras protein. Mol. Cell. Biol. 12 (6), 2606-2615.

Coxon, J.P., Oades, G.M., Kirby, R.S., Colston, K.W., 2004. Zoledronic acid induces apoptosis and inhibits adhesion to mineralized matrix in prostate cancer cells via inhibition of protein prenylation. BJU Int. 94 (1), 164-170.

Crul, M., de Klerk, G.J., Beijnen, J.H., Schellens, J.H.M., 2001. Ras biochemistry and farnesyl transferase inhibitors: a literature survey. Anti-Cancer Drug 12 (3) $163-184$

DeBose-Boyd, R.A., 2008. Feedback regulation of cholesterol synthesis: sterol accelerated ubiquitination and degradation of HMG CoA reductase. Cell Res. 18 (6), 609-621.

Denoyelle, C., Vasse, M., Korner, M., Mishal, Z., Ganne, F., Vannier, J.P., et al., 2001. Cerivastatin, an inhibitor of HMG-CoA reductase, inhibits the signaling pathways involved in the invasiveness and metastatic properties of highly invasive breast cancer cell lines: an in vitro study. Carcinogenesis 22 (8), 1139-1148.

Denoyelle, C., Hong, L., Vannier, J.P., Soria, J., Soria, C., 2003. New insights into the actions of bisphosphonate zoledronic acid in breast cancer cells by dual RhoAdependent and -independent effects. Br. J. Cancer 88 (10), 1631-1640.

Di Chiara, A., Vanuzzo, D., 2009. Does surveillance impact on cardiovascular prevention? Eur. Heart J. 30 (9), 1027-1029.

Du, W., Lebowitz, P.F., Prendergast, G.C., 1999. Cell growth inhibition by farnesyl transferase inhibitors is mediated by gain of geranylgeranylated RhoB. Mol. Cell. Biol. 19 (3), 1831-1840.

Ellenbroek, S.I., Collard, J.G., 2007. Rho GTPases: functions and association with cancer. Clin. Exp. Metastasis 24 (8), 657-672.

Farahani, E., Patra, H.K., Jangamreddy, J.R., Rashedi, I., Kawalec, M., Rao Pariti, R.K., et al., 2014. Cell adhesion molecules and their relation to (cancer) cell stemness. Carcinogenesis 35 (4), 747-759.

Fernandez, C., Lobo Md Mdel, V., Gomez-Coronado, D., Lasuncion, M.A., 2004. Cholesterol is essential for mitosis progression and its deficiency induces polyploid cell formation. Exp. Cell Res. 300 (1), 109-120.
Fernandez, A., Huggins, I.J., Perna, L., Brafman, D., Lu, D., Yao, S., et al., 2014. The WNT receptor FZD7 is required for maintenance of the pluripotent state in human embryonic stem cells. Proc. Natl. Acad. Sci. U.S.A. 111 (4), 1409-1414.

Fleisch, H., 1991. Bisphosphonates. Pharmacology and use in the treatment of tumour-induced hypercalcaemic and metastatic bone disease. Drugs 42 (6), 919-944.

Fleisch, H., 1998. Bisphosphonates: mechanisms of action. Endocrine Rev. 19 (1), 80-100.

Frick, M., Dulak, J., Cisowski, J., Jozkowicz, A., Zwick, R., Alber, H., et al., 2003. Statins differentially regulate vascular endothelial growth factor synthesis in endothelial and vascular smooth muscle cells. Atherosclerosis 170 (2), 229-236.

Fritz, G., 2009. Targeting the mevalonate pathway for improved anticancer therapy. Curr. Cancer Drug Targets 9 (5), 626-638.

Gaist, D., Hallas, J., Friis, S., Hansen, S., Sorensen, H.T., 2014. Statin use and survival following glioblastoma multiforme. Cancer Epidemiol. 38 (6), 722-727.

Gauthaman, K., Fong, C.Y., Bongso, A., 2009. Statins, stem cells, and cancer. J. Cell. Biochem. 106 (6), 975-983.

Ghavami, S., Yeganeh, B., Stelmack, G.L., Kashani, H.H., Sharma, P., Cunnington, R., et al., 2012a. Apoptosis, autophagy and ER stress in mevalonate cascade inhibition-induced cell death of human atrial fibroblasts. Cell Death Dis. 3, e330.

Ghavami, S., Mutawe, M.M., Schaafsma, D., Yeganeh, B., Unruh, H., Klonisch, T. et al., 2012b. Geranylgeranyl transferase 1 modulates autophagy and apoptosis in human airway smooth muscle. Am. J. Physiol. Lung Cell. Mol. Physiol. 302 (4), L420-L428.

Ghavami, S., Sharma, P., Yeganeh, B., Ojo, O.O., Jha, A., Mutawe, M.M., et al., 2014 Airway mesenchymal cell death by mevalonate cascade inhibition: integration of autophagy, unfolded protein response and apoptosis focusing on Bcl2 family proteins. Biochim. Biophys. Acta 1843 (7), 1259-1271.

Goldstein, J.L., Brown, M.S., 1990. Regulation of the mevalonate pathway. Nature 343 (6257), 425-430.

Gonen, N., Assaraf, Y.G., 2012. Antifolates in cancer therapy: structure, activity and mechanisms of drug resistance. Drug Resist. Updates 15 (4), 183-210 (Reviews and commentaries in antimicrobial and anticancer chemotherapy.)

Graaf, M.R., Richel, D.J., van Noorden, C.J., Guchelaar, H.J., 2004a. Effects of statins and farnesyltransferase inhibitors on the development and progression of cancer. Cancer Treat. Rev. 30 (7), 609-641.

Graaf, M.R., Beiderbeck, A.B., Egberts, A.C., Richel, D.J., Guchelaar, H.J., 2004b. The risk of cancer in users of statins. J. Clin. Oncol. 22 (12), 2388-2394 (Official journal of the American Society of Clinical Oncology).

Gunde-Cimerman, N., Cimerman, A., 1995. Pleurotus fruiting bodies contain the inhibitor of 3-hydroxy-3-methylglutaryl-coenzyme A reductase-lovastatin. Exp. Mycol. 19 (1), 1-6.

Haeffner, E.W., Hoffmann, C.J., Stoehr, M., Scherf, H., 1984. Cholesterol-induced growth stimulation, cell aggregation, and membrane properties of ascites tumor cells in culture. Cancer Res. 44 (6), 2668-2676.

Hall, A., 1998. Rho GTPases and the actin cytoskeleton. Science 279 (5350), 509-514.

Hamilton, S.R., Fard, S.F., Paiwand, F.F., Tolg, C., Veiseh, M., Wang, C., et al., 2007. The hyaluronan receptors CD44 and Rhamm (CD168) form complexes with ERK1,2 that sustain high basal motility in breast cancer cells. J. Biol. Chem. 282 (22), 16667-16680

Hancock, J.F., Magee, A.I., Childs, J.E., Marshall, C.J., 1989. All Ras proteins are polyisoprenylated but only some are palmitoylated. Cell 57 (7), 1167-1177.

Harvey, K.F., Zhang, X., Thomas, D.M., 2013. The Hippo pathway and human cancer. Nat. Rev. Cancer 13 (4), 246-257.

Hinson, D.D., Chambliss, K.L., Toth, M.J., Tanaka, R.D., Gibson, K.M., 1997 Post-translational regulation of mevalonate kinase by intermediates of the cholesterol and nonsterol isoprene biosynthetic pathways. J. Lipid Res. 38 (11), 2216-2223

Holdgate, G.A., Ward, W.H., McTaggart, F., 2003. Molecular mechanism for inhibition of 3-hydroxy-3-methylglutaryl CoA (HMG-CoA) reductase by rosuvastatin. Biochem. Soc. Trans. 31 (Pt 3), 528-531.

Huang, M., Prendergast, G.C., 2006. RhoB in cancer suppression. Histol. Histopathol. 21 (2), 213-218

Iishi, H., Tatsuta, M., Baba, M., Yano, H., Sakai, N., Uehara, H., et al., 2000. Ras p21 Isoprenylation inhibition induces flat colon tumors in Wistar rats. Dis. Colon Rectum 43 (1), 70-75

Istvan, E.S., 2002. Structural mechanism for statin inhibition of 3-hydroxy-3methylglutaryl coenzyme A reductase. Am. Heart J. 144 (6 Suppl), S27-S32.

Istvan, E., 2003. Statin inhibition of HMG-CoA reductase: a 3-dimensional view. Atherosclerosis Suppl. 4 (1), 3-8.

Jackson, J.H., Cochrane, C.G., Bourne, J.R., Solski, P.A., Buss, J.E., Der, C.J., 1990. Farnesol modification of Kirsten-Ras Exon 4b-protein is essential for transformation. Proc. Natl. Acad. Sci. U.S.A. 87 (8), 3042-3046.

Jain, M.V., Jangamreddy, J.R., Grabarek, J., Schweizer, F., Klonisch, T., CieslarPobuda, A., et al., 2015. Nuclear localized Akt enhances breast cancer stem-like cells through counter-regulation of p21(Waf1/Cip1) and p27(kip1). Cell Cycle (Georgetown, Tex) 14 (13), 2109-2120.

Jangamreddy, J.R., Ghavami, S., Grabarek, J., Kratz, G., Wiechec, E., Fredriksson, B.A., et al., 2013. Salinomycin induces activation of autophagy, mitophagy and affects mitochondrial polarity: differences between primary and cancer cells. Biochim. Biophys. Acta 1833 (9), 2057-2069.

Jaswal, R.S., Kaushal, R.K., Goel, A., Pathania, K., 2003. Role of C-reactive protein in deciding duration of antibiotic therapy in neonatal septicemia. Indian Pediatr. 40 (9), 880-883.

Jick, H., Zornberg, G.L., Jick, S.S., Seshadri, S., Drachman, D.A., 2000. Statins and the risk of dementia. Lancet 356 (9242), 1627-1631. 
Kalluri, R., Weinberg, R.A., 2009. The basics of epithelial-mesenchymal transition. J. Clin. Investig. 119 (6), 1420-1428.

Kamai, T., Yamanishi, T., Shirataki, H., Takagi, K., Asami, H., Ito, Y., et al., 2004. Overexpression of RhoA, Rac1, and Cdc42 GTPases is associated with progression in testicular cancer. Clin. Cancer Res. 10 (14), 4799-4805.

Kato, K., Cox, A.D., Hisaka, M.M., Graham, S.M., Buss, J.E., Der, C.J., 1992. Isoprenoid addition to Ras protein is the critical modification for its membrane association and transforming activity. Proc. Natl. Acad. Sci. U.S.A. 89 (14), 6403-6407

Katoh, M., 2005. WNT/PCP signaling pathway and human cancer (review). Oncol. Rep. 14 (6), 1583-1588.

Keep, N.H., Barnes, M., Barsukov, I., Badii, R., Lian, L.Y., Segal, A.W., et al., 1997. A modulator of rho family $G$ proteins, rhoGDI, binds these $G$ proteins via an immunoglobulin-like domain and a flexible N-terminal arm. Structure 5 (5), 623-633.

Keyomarsi, K., Sandoval, L., Band, V., Pardee, A.B., 1991. Synchronization of tumor and normal cells from G1 to multiple cell cycles by lovastatin. Cancer Res. 51 (13), 3602-3609.

Koo, B.S., Lee, S.H., Kim, J.M., Huang, S., Kim, S.H., Rho, Y.S., et al., 2014. Oct4 is a critical regulator of stemness in head and neck squamous carcinoma cells. Oncogene 34, 2317-2324.

Kotwal, S., Dhar, M.K., Kour, B., Raj, K., Kaul, S., 2013. Molecular markers unravel intraspecific and interspecific genetic variability in Plantago ovata and some of its wild allies. J. Genet. 92 (2), 293-298.

Koyuturk, M., Ersoz, M., Altiok, N., 2004. Simvastatin induces proliferation inhibition and apoptosis in C6 glioma cells via c-jun N-terminal kinase. Neurosci. Lett. 370 (2-3), 212-217.

Lebowitz, P.F., Du, W., Prendergast, G.C., 1997. Prenylation of RhoB is required for its cell transforming function but not its ability to activate serum response elementdependent transcription. J. Biol. Chem. 272 (26), 16093-16095.

Lee, M.H., Cho, Y.S., Han, Y.M., 2007. Simvastatin suppresses self-renewal of mouse embryonic stem cells by inhibiting RhoA geranylgeranylation. Stem Cells (Dayton, Ohio) 25 (7), 1654-1663.

Lee, S.H., Koo, B.S., Kim, J.M., Huang, S., Rho, Y.S., Bae, W.J., et al., 2014. Wnt/betacatenin signalling maintains self-renewal and tumourigenicity of head and neck squamous cell carcinoma stem-like cells by activating Oct4. J. Pathol. 234 (1), 99-107.

Lennernas, H., Fager, G., 1997. Pharmacodynamics and pharmacokinetics of the HMG-CoA reductase inhibitors. Similarities and differences. Clin. Pharmacokinet. 32 (5), 403-425.

Li, W., Zhu, T., Guan, K.L., 2004. Transformation potential of Ras isoforms correlates with activation of phosphatidylinositol 3-kinase but not ERK. J. Biol. Chem. 279 (36), 37398-37406.

Li, L., Ying, J., Li, H., Zhang, Y., Shu, X., Fan, Y., et al., 2012. The human cadherin 11 is a pro-apoptotic tumor suppressor modulating cell stemness through Wnt/beta-catenin signaling and silenced in common carcinomas. Oncogene 31 (34), 3901-3912.

Liu, A.X., Cerniglia, G.J., Bernhardt, E.J., Prendergast, G.C., 2001. RhoB is required to mediate apoptosis in neoplastically transformed cells after DNA damage. Proc. Natl. Acad. Sci. U.S.A. 98 (11), 6192-6197.

Liu, J.P., Yang, M., Liu, Y.X., Wei, M., Grimsgaard, S., 2006. Herbal medicines for treatment of irritable bowel syndrome. Cochrane Database Syst. Rev. 25 (1), CD004116.

Livney, Y.D., Assaraf, Y.G., 2013. Rationally designed nanovehicles to overcome cancer chemoresistance. Adv. Drug Delivery Rev. 65 (13-14), $1716-1730$

Lobell, R.B., Omer, C.A., Abrams, M.T., Bhimnathwala, H.G., Brucker, M.J., Buser, C.A., et al., 2001. Evaluation of farnesyl: protein transferase and geranylgeranyl: protein transferase inhibitor combinations in preclinical models. Cancer Res. 61 (24), 8758-8768.

Ma, P.T., Gil, G., Südhof, T.C., Bilheimer, D.W., Goldstein, J.L., Brown, M.S., 1986. Mevinolin, an inhibitor of cholesterol synthesis, induces mRNA for low density lipoprotein receptor in livers of hamsters and rabbits. Proc. Natl. Acad. Sci. U.S.A. 83 (21), 8370-8374.

Maddika, S., Panigrahi, S., Wiechec, E., Wesselborg, S., Fischer, U., Schulze-Osthoff, K., et al., 2009. Unscheduled Akt-triggered activation of cyclin-dependent kinase 2 as a key effector mechanism of apoptin's anticancer toxicity. Mol. Cell. Biol. 29 (5), 1235-1248.

Magnusson, K., Appelqvist, H., Cieslar-Pobuda, A., Wigenius, J., Karlsson, T., Los, M.J., et al., 2015. Differential vital staining of normal fibroblasts and melanoma cells by an anionic conjugated polyelectrolyte. Cytometry A 87 (3), 262-272 (The journal of the International Society for Analytical Cytology).

Maltese, W.A., Defendini, R., Green, R.A., Sheridan, K.M., Donley, D.K., 1985. Suppression of murine neuroblastoma growth in vivo by mevinolin, a competitive inhibitor of 3-hydroxy-3-methylglutaryl-coenzyme A reductase. J. Clin. Investig. 76 (5), 1748-1754.

Maurer-Stroh, S., Eisenhaber, F., 2005. Refinement and prediction of protein prenylation motifs. Genome Biol. 6 (6), R55.

Maurer-Stroh, S., Washietl, S., Eisenhaber, F., 2003. Protein prenyltransferases. Genome Biol. 4 (4), 212.

Melchior, K., Weiss, J., Zaehres, H., Kim, Y.M., Lutzko, C., Roosta, N., et al., 2008. The WNT receptor FZD7 contributes to self-renewal signaling of human embryonic stem cells. Biol. Chem. 389 (7), 897-903.

Mijimolle, N., Velasco, J., Dubus, P., Guerra, C., Weinbaum, C.A., Casey, P.J., et al., 2005. Protein farnesyltransferase in embryogenesis, adult homeostasis, and tumor development. Cancer Cell 7 (4), 313-324.
Misra, J., Mohanty, S.T., Madan, S., Fernandes, J.A., Ebetino, F.H., Graham, R., et al, 2015. Zoledronate attenuates accumulation of DNA damage in mesenchymal stem cells and protects their function. Stem Cells (Dayton, Ohio), http://dx.doi. org/10.1002/stem.2255.

Monkkonen, H., Auriola, S., Lehenkari, P., Kellinsalmi, M., Hassinen, I.E., Vepsalainen, J., et al., 2006. A new endogenous ATP analog (ApppI) inhibits the mitochondrial adenine nucleotide translocase (ANT) and is responsible for the apoptosis induced by nitrogen-containing bisphosphonates. Br. J. Pharmacol. 147 (4) 437-445.

Naidoo, J., Drilon, A., 2016. KRAS-mutant lung cancers in the era of targeted therapy. Adv. Exp. Med. Biol. 893, 155-178.

Novelli, G., D’Apice, M.R., 2012. Protein farnesylation and disease. J. Inherit. Metab. Dis. 35 (5), 917-926.

Panini, S.R., Schnitzerpolokoff, R., Spencer, T.A., Sinensky, M., 1989. Sterolindependent regulation of 3-hydroxy-3-methylglutaryl-Coa reductase by mevalonate in Chinese-Hamster ovary cells-magnitude and specificity. J. Biol. Chem. 264 (19), 11044-11052.

Papapoulos, S.E., 2008. Bisphosphonates: how do they work? Best Pract. Res. Clin. Endocrinol. Metab. 22 (5), 831-847.

Paterson, H.F., Self, A.J., Garrett, M.D., Just, I., Aktories, K., Hall, A., 1990. Microinjection of recombinant p21rho induces rapid changes in cell morphology. J. Cell Biol. 111 (3), 1001-1007.

Perona, R., Montaner, S., Saniger, L., SanchezPerez, I., Bravo, R., Lacal, J.C., 1997. Activation of the nuclear factor-kappa B by Rho, CDC42, and Rac-1 proteins. Genes Dev. 11 (4), 463-475.

Peterson, Y.K., Kelly, P., Weinbaum, C.A., Casey, P.J., 2006. A novel protein geranylgeranyltransferase-I inhibitor with high potency, selectivity, and cellular activity. J. Biol. Chem. 281 (18), 12445-12450.

Philips, M.R., Cox, A.D., 2007. Geranylgeranyltransferase I as a target for anti-cancer drugs. J. Clin. Investig. 117 (5), 1223-1225.

Piccolo, S., Cordenonsi, M., Dupont, S., 2013. Molecular pathways: YAP and TAZ take center stage in organ growth and tumorigenesis. Clin. Cancer Res. 19 (18), 4925-4930.

Piccolo, S., Dupont, S., Cordenonsi, M., 2014. The biology of YAP/TAZ: hippo signaling and beyond. Physiol. Rev. 94 (4), 1287-1312.

Pich, C., Teiti, I., Rochaix, P., Mariame, B., Couderc, B., Favre, G., et al., 2013. Statins reduce melanoma development and metastasis through MICA overexpression. Front. Immunol. 4, 62.

Pille, J.Y., Denoyelle, C., Varet, J., Bertrand, J.R., Soria, J., Opolon, P., et al., 2005. AntiRhoA and anti-RhoC siRNAs inhibit the proliferation and invasiveness of MDA MB-231 breast cancer cells in vitro and in vivo. Mol. Ther. 11 (2), 267-274.

Ramos, A., Camargo, F.D., 2012. The Hippo signaling pathway and stem cell biology. Trends Cell Biol. 22 (7), 339-346.

Rao, S., Lowe, M., Herliczek, T.W., Keyomarsi, K., 1998. Lovastatin mediated G1 arrest in normal and tumor breast cells is through inhibition of CDK2 activity and redistribution of p21 and p27, independent of p53. Oncogene 17 (18), 2393-2402.

Reya, T., Morrison, S.J., Clarke, M.F., Weissman, I.L., 2001. Stem cells, cancer, and cancer stem cells. Nature 414 (6859), 105-111.

Ridley, A.J., Hall, A., 1992. The small GTP-binding protein rho regulates the assembly of focal adhesions and actin stress fibers in response to growth factors. Cell 70 (3), 389-399.

Ridley, A.J., 2004. Rho proteins and cancer. Breast Cancer Res. Treat. 84 (1), 13-19.

Rollano Penaloza, O.M., Lewandowska, M., Stetefeld, J., Ossysek, K., Madej, M., Bereta, J., et al., 2014. Apoptins: selective anticancer agents. Trends Mol. Med. 20 (9), 519-528.

Roskoski Jr., R., 2003. Protein prenylation: a pivotal posttranslational process Biochem. Biophys. Res. Commun. 303 (1), 1-7

Sahai, E., Marshall, C.J., 2002. RHO-GTPases and cancer. Nat. Rev. Cancer 2 (2), 133-142.

Sane, K.M., Mynderse, M., Lalonde, D.T., Dean, I.S., Wojtkowiak, J.W., Fouad, F., et al., 2010. A novel geranylgeranyl transferase inhibitor in combination with lovastatin inhibits proliferation and induces autophagy in STS-26T MPNST cells. J. Pharmacol. Exp. Ther. 333 (1), 23-33.

Sasaki, T., Takai, Y., 1998. The Rho small G protein family Rho GDI system as a temporal and spatial determinant for cytoskeletal control. Biochem. Biophys. Res. Commun. 245 (3), 641-645

Schaefer, E.J., McNamara, J.R., Tayler, T., Daly, J.A., Gleason, J.L., Seman, L.J., et al., 2004 Comparisons of effects of statins (atorvastatin, fluvastatin, lovastatin, pravas tatin, and simvastatin) on fasting and postprandial lipoproteins in patients with coronary heart disease versus control subjects. Am. J. Cardiol. 93 (1), 31-39.

Scheffzek, K., Ahmadian, M.R., Kabsch, W., Wiesmuller, L., Lautwein, A., Schmitz, F., et al., 1997. The Ras-RasGAP complex: structural basis for GTPase activation and its loss in oncogenic Ras mutants. Science 277 (5324), 333-338.

Sebti, S.M., 2005. Protein farnesylation: implications for normal physiology, malignant transformation, and cancer therapy. Cancer Cell 7 (4), 297-300.

Shepherd, J., 2006. Who should receive a statin these days? Lessons from recent clinical trials. J. Intern. Med. 260 (4), 305-319.

Simons, M., Keller, P., Dichgans, J., Schulz, J.B., 2001. Cholesterol and Alzheimer's disease: is there a link? Neurology 57 (6), 1089-1093.

Singh, P., Saxena, R., Srinivas, G., Pande, G., Chattopadhyay, A., 2013. Cholesterol biosynthesis and homeostasis in regulation of the cell cycle. PLoS ONE 8 (3) e58833.

Sjogren, A.K., Andersson, K.M., Liu, M., Cutts, B.A., Karlsson, C., Wahlstrom, A.M., et al., 2007. GGTase-I deficiency reduces tumor formation and improves survival in mice with K-RAS-induced lung cancer. J. Clin. Investig. 117 (5), 1294-1304. 
Smith, F.J.D., Sandilands, A., McLean, W.H.I., 2004. Molecular genetics methods for human intermediate filament diseases. Method Cell Biol. 78, 131-161.

Sorrentino, G., Ruggeri, N., Specchia, V., Cordenonsi, M., Mano, M., Dupont, S., et al., 2014. Metabolic control of YAP and TAZ by the mevalonate pathway. Nat. Cell Biol. 16 (4), 357-366.

Swanson, K.M., Hohl, R.J., 2006. Anti-cancer therapy: targeting the mevalonate pathway. Curr. Cancer Drug Targets 6 (1), 15-37.

Tardoski, S., Ngo, J., Gineyts, E., Roux, J.P., Clezardin, P., Melodelima, D., 2015. Lowintensity continuous ultrasound triggers effective bisphosphonate anticancer activity in breast cancer. Sci. Rep. 5, 16354.

Taylor, S., Spugnini, E.P., Assaraf, Y.G., Azzarito, T., Rauch, C., Fais, S., 2015. Microenvironment acidity as a major determinant of tumor chemoresistance: proton pump inhibitors (PPIs) as a novel therapeutic approach. Drug Resist. Updates 23, 69-78 (Reviews and commentaries in antimicrobial and anticancer chemotherapy).

Taylor, J., 2012. Joint societies CVD Prevention Guidelines launched in May 2012. Eur. Heart J. 33 (13), 1539.

Van Acker, H.H., Anguille, S., Willemen, Y., Smits, E.L., Van Tendeloo, V.F., 2016. Bisphosphonates for cancer treatment: mechanisms of action and lessons from clinical trials. Pharmacol. Ther. 158, 24-40, http://dx.doi.org/10.1016/j. pharmthera.2015.11.008.

van Beek, E.R., Cohen, L.H., Leroy, I.M., Ebetino, F.H., Lowik, C.W., Papapoulos, S.E., 2003. Differentiating the mechanisms of antiresorptive action of nitrogen containing bisphosphonates. Bone 33 (5), 805-811.

Veeman, M.T., Axelrod, J.D., Moon, R.T., 2003. A second canon. Functions and mechanisms of beta-catenin-independent Wnt signaling. Dev. Cell 5 (3), 367-377.

Vega, F.M., Ridley, A.J., 2008. Rho GTPases in cancer cell biology. FEBS Lett. 582 (14) 2093-2101.

Vincan, E., Darcy, P.K., Farrelly, C.A., Faux, M.C., Brabletz, T., Ramsay, R.G., 2007. Frizzled-7 dictates three-dimensional organization of colorectal cancer cell carcinoids. Oncogene 26 (16), 2340-2352.

Vogt, A., Sun, J., Qian, Y., Hamilton, A.D., Sebti, Sd.M., 1997. The geranylgeranyltransferase-I inhibitor GGTI-298 arrests human tumor cells in G0/G1 and induces p21WAF1/CIP1/SDI1 in a p53-independent manner. J. Biol. Chem. 272 (43), 27224-27229.

Wakchoure, S., Merrell, M.A., Aldrich, W., Millender-Swain, T., Harris, K.W., Triozzi, P., et al., 2006. Bisphosphonates inhibit the growth of mesothelioma cells in vitro and in vivo. Clin. Cancer Res. 12 (9), 2862-2868.

Wang, Z., Wu, Y., Wang, H., Zhang, Y., Mei, L., Fang, X., et al., 2014. Interplay of mevalonate and Hippo pathways regulates RHAMM transcription via YAP to modulate breast cancer cell motility. Proc. Natl. Acad. Sci. U.S.A. 111 (1), E89-E98.

Wasik, A.M., Grabarek, J., Pantovic, A., Cieslar-Pobuda, A., Asgari, H.R., BundgaardNielsen, C., et al., 2014. Reprogramming and carcinogenesis-parallels and distinctions. Int. Rev. Cell Mol. Biol. 308, 167-203.

Weis, M., Heeschen, C., Glassford, A.J., Cooke, J.P., 2002. Statins have biphasic effects on angiogenesis. Circulation 105 (6), 739-745.

Whyte, D.B., Kirschmeier, P., Hockenberry, T.N., NunezOliva, I., James, L., Catino, J.J., et al., 1997. K- and N-Ras are geranylgeranylated in cells treated with farnesyl protein transferase inhibitors. J. Biol. Chem. 272 (22), 14459-14464.

Wojtuszkiewicz, A., Raz, S., Stark, M., Assaraf, Y.G., Jansen, G., Peters, G.J., et al., 2016. Folylpolyglutamate synthetase splicing alterations in acute lymphoblastic leukemia are provoked by methotrexate and other chemotherapeutics and mediate chemoresistance. J. Int. Cancer 138, 1645-1656, http://dx.doi.org/10. 1002/ijc.29919.

Wolozin, B., Kellman, W., Ruosseau, P., Celesia, G.G., Siegel, G., 2000. Decreased prevalence of Alzheimer disease associated with 3-hydroxy-3-methyglutaryl coenzyme A reductase inhibitors. Arch. Neurol. 57 (10), 1439-1443.

Yang, W.L., Urano, J., Tamanoi, F., 2000. Protein farnesylation is critical for maintaining normal cell morphology and canavanine resistance in Schizosaccharomyces pombe. J. Biol. Chem. 275 (1), 429-438.

Yeganeh, B., Wiechec, E., Ande, S.R., Sharma, P., Moghadam, A.R., Post, M., et al., 2014. Targeting the mevalonate cascade as a new therapeutic approach in heart disease, cancer and pulmonary disease. Pharmacol. Ther. 143 (1), 87-110.

Yoon, C.H., Hyun, K.H., Kim, R.K., Lee, H., Lim, E.J., Chung, H.Y., et al., 2011. The small GTPase Rac1 is involved in the maintenance of stemness and malignancies in glioma stem-like cells. FEBS Lett. 585 (14), 2331-2338.

Zeman, J., Shipman, K., 1997. Social-contextual influences on expectancies for managing anger and sadness: the transition from middle childhood to adolescence. Dev. Psychol. 33 (6), 917-924.

Zhang, F.L., Casey, P.J., 1996. Protein prenylation: molecular mechanisms and functional consequences. Annu. Rev. Biochem. 65, 241-269.

Zhang, S.J., Han, J.H., Sells, M.A., Chernoff, J., Knaus, U.G., Ulevitch, R.J., et al., 1995. Rho-family Gtpases regulate P38 mitogen-activated protein-kinase through the downstream mediator Pak1. J. Biol. Chem. 270 (41), 23934-23936.

Zhao, X., Hu, X., 2015. Dosing of zoledronic acid with its anti-tumor effects in breast cancer. J. Bone Oncol. 4 (3), 98-101.

Zhitomirsky, B., Assaraf, Y.G., 2016. Lysosomes as mediators of drug resistance in cancer. Drug Resist. Updates 24, 23-33, http://dx.doi.org/10.1016/j.drup.2015. 11.004. 